\title{
MODALITÉS VERBALES DU SUBJONCTIF À LA LUMIÈRE D'UNE ANALYSE CONTRASTIVE FONCTIONNELLE DANS LES LANGUES FRANGAISE ET SLOVÈNE*
}

1. La langue francaise est caractérisée par 1'existence $d$ 'un paradigme verbal spécial, le subjonctif, que les grammaires franqaises définissent, en géneral, comme mode de 1 'incertitude, du doute, parce qu'il "présente 1 'action comme simplement envisagée, comme n'étant pas placée sur le plan de la réalite" (Grevisse 1964, par. 613).

Ce travail-ci est né de la conscience qu'un locuteur slovène, qui dans sa langue ne connâ̂t pas un paradigme verbal correspondant au subjonctif français, mais seulement une série de moyens partiels, est constamment menace d'appliquer quand il parle francais le système verbal qui vaut dans sa propre langue.

2.1 Les linguistes adoptent, envers ce paradigme verbal, des positions assez variées.

* La Faculte des Lettres de l'Univeristé de Ljubljana avait accepté le 20 octobre 1980 le thème suivant "Modalités verbales du subjonctif à la lumière de l'analyse contrastive frangais-slovène", proposé par Mme Darja Globevnik, alors assistante auprès de I Académie slovene des sciences et des Arts, comme sujet de sa thèse. Nommé directeur d'études, $j$ 'étais donc chargé de suivre ce travail.

Mme Globevnik a terminé sa these et l'a présentée en slovène au mois de février 1983. Mais la disparition tragique de Mme Globevnik a interrompu ce travail. La thèse n'a pas pu être soutenue.

Afin que les résultats de cette recherche et les idées théoriques qui avaient été à la base de son travail puissent être accessibles à tous ceux qui s'intéressent à l'étude des modes en francais, nous avons tenu à publier l'essentiel de cette thèse dans le volume de Linguistica, consacré plus particulièrement aux premières oeuvres des jeunes chercheurs débutant dans la carrière scientifique. En préparant le texte pour cette publication, ma tâche principale a été de rendre le plus scrupuleusement possible les ideses de l'auteur, en dépit des coupures nécessaires. - Mitja skubic 
La majorite d'entre eux definissent le subjonctif comme un signe linguistique qui unit une expression donné avec un contenu donné. L 'apparition du subjonctif est conditionnée par ce contenu. On peut concevoir ces theories comme des théories sémantiques. Elles voient dans le subjonctif francais 1 'expression d'une notion fondamentale, à la rigueur deux notions, mais rarement plus. Elles cherchent, en effet, à réduire les emplois du subjonctif à un seul dénominateur ou deux, ce qui devrait représenter la qualité essentielle; c'est à cause de cela, que cette qualité essentielle est souvent assez vaguement définie.

D'après ces théries, le subjonctif franqais et roman serait:

le mode de la subjectivité (van der Molen 1923)

un mode affectif, subjectif (Dauzat 1947)

proprement le mode de I'énergie psychique (Wartburg-zumthor 1947), (Le Bidois-Le Bidois 1947)

Une réserve, un recul en face de l'affirmation (Hanse 1960)

essentiellement un mouvement d'âme (Mauger 1968)

une appréciation affective (Pohl s. d.)

ein direkter Ausdruck der Nichtwirklichkeit (Haas 1909)

die Spiegelung des Gefülhs der Unsicherheit (Soltmann 1914)

1 idée énoncée. est considérée comme possible ou deuteuse (Gramm. de 1'Académie 1932)

die Realitätsfrage vollig ignoriert (Kalepky 1928)

indifférent à la réalité... neutre (de Poerck 1950)

le mode de la prise en pensée (Regula 1957)

un procès simplement envisage par 1 'esprit (Grevisse 1964)

der Modus des.. virtuel Betrachten (Schifko 1967)

réduit, aujourd'hui, à exprimer 1 action simplement envisagée par I'esprit (Brunot-Bruneau 1969)

une pure et simple conception de l'esprit (Martinet 1979)

le mode du temps amorphe (Guillaume 1929)

Timeless... contrasts by absence of any reference to time (Hall 1948)

le mode du temps amorphe (Moignet 1959) 
le fait relaté $\mathrm{n}$ 'est pas entièrement actualisé (Imbs 1953)

toutes les fois que $n$ 'est pas exprimée une nouvelle affirmation (Damourette et Pichon 1911-36)

suspension de 1 affirmation (Togeby 1953)

the mark of non-assertion (Freeman 1974)

D-autres voient plus d une qualité essentielle et surtout l'incertitude, la volonté, le sentiment, I appréciation du réel (ou du possible); ainsi Gramm. Larousse 1964: "le mode ... pour aprécier la réalisation ou les possibilités de réalisation de 1 "action". M. Cohen 1965 arrive à distinguer plus de vingt qualités, commandement, souhait, supposition, obligation, appréciation, réserve, regret etc.

2.2 Ensuite, il $\mathrm{y}$ a des théories syntaxiques: le subjonctif ne serait qu'une variante de 1 'indicatif. Pour Togeby 1965 c'est un mode extensif-intensif, pour Ruwet 1965 "n'est pas un mode. Étant substitué à un élément dominé pax le temps, il acquiert dans la structure syntagmatique dérivée la catégorie syntaxique de cet elément; autrement dit, il est un temps." Le subjonctif est donc un paradigme sans valeur sémantique propre.

2.3 Enfin, les théories sémantico-syntaxiques voient dans le subjonctif roman en même temps un signe linguistique et une variante morphologique de l'indicatif; d'autres, qu'on pourrait classer sémantiques + syntaxiques, voient dans ce paradigme un signe linguistique avec quelques caractéristiques syntaxiques.

3.1 L étude contrastive permet de prévoir que toutes ces theories sur le subjonctif francais ne peuvent pas avoir la même validité.

La série syntaxique des théories, d 'après laquelle le subjonctif $n$ 'est qu'une variante de 1 indicatif, explique le subjonctif comme une forme plus au moins accidentelle. Il est impossible de croire qu "à une forme linguistique, exclusivement "forme", dans une langue (soient comme exemple verbes "irréguliers" ou "formes irrégulières" du pluriel des substantifs) corresponde dans une autre langue une structure clairement délimitée et homogène. Un tel point de départ, se basant exclusivement sur des critères formels, à priori ne donne pas beau- 
coup de possibilités pour obtenir des résultats positifs.

Les théories sémantiques, d'après lesquelles le subjonctif est un signe linguistique, definissent le subjonctif comme un paradigme verbal non-accidentel et qui apparaît conforme a son contenu sémantique. Il est plus probable que le contenu sémantique d'un signe linguistique dans une langue ait une structure, plus au moins nettement délimitée, dans 1 'autre. Un tel point de départ, basé sur la réalité sémantique, donne par conséquent à 1 'analyse contrastive un meilleur espoir de résultat positif. Une théorie sémantique est plus naturelle dans la recherche contrastive: I'élement sémantique est plus important que 1 'élement formel, autrement dit, on traduit par le sens et non par les formes.

3.2 Dans I analyse contrastive on attend la mise en évidence de deux points:

a) le subjonctif franqais doit être sémantiquement analysé avec précision;

b) en I analysant, il faut mettre en relief les traits qui montrent les différences structurales entre les deux langues.

Les conditions fondamentales de la recherche sont la précision de la description, c'est-à-dire, la non contradiction avec les faits de langue et la conformité méthodologique de I'élaboration.

Une thérie sémantique tend à ce que la description soit autant que possible exacte. Autrement dit, elle ne doit pas venir au conflit avec la réalité linguistique, avec les faits de langue. En outre, il est souhaitable qu'un phénomène linguistique soit décrit de la manière le plus simple possible et intuitivement convenable.

La majeure partie des théories sémantiques du subjonctif roman délimitent la nature sémantique du subjonctif de points de vue tres différents; pourtant, elles lui attribuent une seule qualité essentielle. Il y en a bien peu qui lui attribuent deux qualités essentielles ou plus, et encore, ces traits distinctifs ne sont pas présentés comme conditionnant une structure. Les theories sémantiques qui cherchent a réduire les dé- 
nominateurs communs de l'emploi du subjonctif si possible à un, sont, formellement, assez simples, sobres et par cela même élégantes; seulement, elles sont nécessairement assez abstraites, et, compte tenu de la varieté des emplois du subjonctif, elles sont trop schématiques. La réduction proposée des valeurs du subjonctif serait justifiée si la definition, obtenue par un procédé semblable, épuisait sa caractéristique essentielle et $n$ 'entrait pas en contradiction avec les faits de langue.

Les expériences de ces théories sémantiques, notamment celles à un seul dénominateur commun, sont trop genérales et abstraites: elles ne disent pas grand chose sur la vraie valeur de ce paradigme verbal et, par conséquent, restent invérifiables (le subjonctif serait "le mode de la subjectivité", "de 1 'énergie psychique", "de la perspective").

Plus articulées, ces theories se trouvent trop souvent en contradiction avec la réalité linguistique. Citons par exemple la theorie qui définit le subjonctif comme le mode du doute: sa contradiction avec les faites de langue est éclatante dans les cas où il n'y a pas de traces de doute; au contraire, la réalisation de l'action, exprimée par le verbe au subjonctif, est postulée: JE SUIS CONTENT QU'IL SOIT VENU.

La théorie sémantique qui définit le subjonctif comme le mode de la volonté se trouve en contradiction avec la réalité dans tous les cas où aucune nuance de volonté $\mathrm{n}^{\prime}$ est présentée: JE SUIS CONTENT QU'IL SOIT VENU; JE DOUTE QU'IL SOIT VENU.

La théorie sémantique qui voit le subjonctif comme le mode d'un temps amorphe est contredite par les cas où la sphère temporelle est nettement exprimée; ainsi, pour l'antéxiorité JE SUIS CONTENT QU'IL AIT AGIT AINSI.

La theorie sémantique qui definit le subjonctif comme le mode d'une non-participation à la situation donnée est contredite par les cas où la situation temporelle est bien délimitée: JE SUIS CONTENT QU'IL SOIT VENU ICI HIER SOIR.

4. Il s'agit d'un sens de réalisme, c'est-à-dire de préciser le degré jusqu'auquel une theorie semble encore valable, malgré les déviations possibles. Il est plus sage d'accepter 
une généralisation de la signification, si celle-ci n'est contredite que par un nombre insignifiant $d$ " "exceptions" et surtout si de telles exceptions sont structurellement liees entre elles, que de s'en tenir à une généralisation dans laquelle on constate toute une série de déviations. En déterminer la limite est, certes, une operation subjective.

Les theories sémantiques qui admettent plusieurs qualités comme essentielles pour $I^{\prime}$ analyse des valeurs du subjonctif sont plus proches de la réalité des faits de langue. Néanmoins, une theorie doit respecter le principe d'une certaine simplicité, de l'économie, ce qui oblige le chercheur à opérer avec un nombre le plus réduit possible de règles de description.

5. Ensuite, une theorie sémantique n'est méthodologiquement soutenable que si elle articule le procéde de la description de telle sorte qu'il puisse être

a) vérifiable, c'est-à-dire, qu'il permette de reconstituer le cheminement de la description par lequel le chercheur est arrivé à classer les phénomenes;

b) utilisable, c'est-à-dire que la description du matériel linguistique puisse être utilisée par n'importe quel chercheur, et pas seulement par le descripteur.

De ce point de vue, les théories sémantiques présentées jusqu' à maintenant, semblent non concordantes et peu satisfaisantes. L'origine de cette carence est liée à la nature même de ces théories. Leur présentations du subjonctif sont intéressantes et éclairent certes des aspects importants de l'apparition de ce paradigme verbal, mais un reproche commun peut être fait à chacune de ces theories: elles sont trop subjectives, basées sur l'intuition du chercheur qui $n^{\prime}$ accorde aucune attention à la structure. Il en résulte que les étapes de la description qui mènent vers les différentes classifications restent pratiquement inverifiables et les théries, par conséquent, ne permettent pas une discussion féconde à partir de critères apparemment si empiriques.

Pour illustrer cette conviction, il suffit d'examiner les diverses classification du verbe CRAINDRE quand il régit une 
subordonnée, par ex. JE CRAINS QU'IL NE VIENNE.

Grevisse 1964, par. 997-1001, classifie les verbes qui régissent les subordonnées complétives dans la catégorie des verbes impersonnels, des verbes d'opinion ou de perception, des verbes de volonik, des verbes de sentiment (et dans cette dernière catégorie se trouve aussi CRAINDRE).

Gougenheim 1930 les classe en verbes de supposition, en verbes de volonté, désir et en verbes affectifs (et parmi ces derniers CRAINDRE).

Nordhal 1969 distingue trois spheres sémantiques: Ze systeme dubitatif, les volitifs, les subjectifs; CRAINDRE fait partie de ces derniers.

CRAINDRE est classé comme verbe de volonté par clédat, par Freeman et par la Grammaire Larousse. Par contre, Lewit 1968 le classe dans la catégorie de doute et Schogt 1968 dans celle de l'engagement subjectif et en même temps postuzé-hypothétique.

Il est difficile à croire que tous ces classements soient sémantiquement convenables. La classification, évidemment, est intuitive.

On pourrait citer maints exemples; qu'il suffise d'en mentionner encore un. La subordonnée relative avec le subjonctif qui a comme antécédent un superlatif ou une expression équivalente exprime

I'incertitude (Lerch 1919),

La certitude (Regula 1958),

I'appréciation subjective ou doute (Gramm. Larousse)

une expression subjectivement formée ou mentalement conGue (Grevisse et Martinet)

Plus encore, le point de vue des auteurs est différent selon qu'ils jugent que l'emploi du subjonctif renforce la déclaration (Soltmann, Carlsson) ou bien l'attenue (Grevisse, Martinet, Schifko, Togeby).

Il s'ensuit qu'une description basée exclusivement sur des données sémantiques (et qui reste intuitive) n'est pas satisfaisante méthodologiquement. Il faut appliquer une théorie sémantique et syntaxique.

6. Dans ce travail, on part d'une théorie sémantico-synta- 
xique. Elle a l'avantage d'interpoler entre l'intuition du chercheur et les matériaux qu'il doit analyser et classifier une étape interméiaire, ce qui diminue, et de beaucoup, la subjectivite. Pour une description exacte du subjonctif frangais il nous faut

a) une description convenable de la sémantique du subjonctif;

b) I'introduction des éléments sémantiques, importants pour l'analyse contrastive.

L'analyse contrastive accompagnera l'analyse des matériaux qu'offrent les textes en franqais; plus encore, la base de ce travail a été justement l'analyse contrastive entre certains textes en français et en slovène. Dans le désir d'utiliser des concepts sémantiques assez palpables, on a réduit les valeurs essentielles du subjonctif français (et il ne semble pas qu'une ultérieure réduction soit possible) à trois: volonté, doute, commentaire.

En soi, ces valeurs posées comme principes ne sont pas une nouveauté: elles sont présentes, ou presque, dans la classification des modalités dans F. Brunot (suivi de Ch. Bally) : volonté, appréciation/jugement, sentiment. Ces principes se retrouvent, quoique séparés, dans les diverses theories sémantiques. Les théories syntaxiques mêmes les connaissent. Par exemple J. Schmitt-Jensen 1970 dans sa théorie definit le subjonctif en italien comme le mode de la dépendance, mais, pour les complétives, il repartit les verbes qui régissent de telles subordonnés en trois catégories: volonté, désir, doute-incertitude.

pourtant, notre travail se différencie des travaux précédents sur les points suivants:

a) Tous les auteurs qui appliquent une tripartition sémantique, l'appliquent seulement aux subordonnées complétives, tandis qu'ils déclarent comme nature essentielle du subjonctif une seule dimension sémantique. Ici, par contre, l'analyse sémantique est primaire, c'est-à-dire, on postule que la théorie du subjonctif est applicable à toutes les subordonnées où on l'utilise, et pas seulement aux complétives. 
b) La deuxième différence consiste dans la délimination du domaine affectif, subjectif. On ne discerne guère dans les faits caractéristiques de ce domaine, I'expression des sentiments et de la subjectivité, mais plutôt le commentaire d'un fait certain. Une telle delimitation est rendue nécessaire par les exigences de précision dans la description du subjonctif français (elle englobe aussi le subjonctif qui apparâtt après des expressions sentimentalement incolores comme C'EST NORMAL, C'EST NATUREL, etc.); aussi, est-elle justifié par l'analyse contrastive qui montre, dans les deux langues comparées, deux systèmes différents.

c) La différence méthodologique, elle aussi, est essentielle. Toutes les descriptions du subjonctif francais se basent sur 1'intuition: les auteurs ne délimitent pas les domaines fondamentaux par des indicateurs systématiques, palpables; ce qui entraine de grandes différences dans le classement du matériel. Ici, au contraire, on part d'une theorie sémantique + syntaxique: on cherchera à delimiter tout d'abord les differents domaines et cela à partir d'une description précise, c'est-à-dire, en se basant sur des critères structuraux.

\subsection{Formation des parallèles structurales}

Les domaines sémantiques: volonté, doute, commentaire une fois établis, nous les déterminerons à l'aide des parallèles structurales.

Le subjonctif étant essentiellement le mode de la subordonnée (et les passages des indépendantes sont facilement analysables avec les mêmes procédés, on analyse toujours la situation proposition principale - proposition subordonnée.

Les parallèles structurales doivent se soumettre aux conditions suivantes:

elles doivent être

a) sensees et intuitivement naturelles;

b) applicables à tous les types de subordonnées subjonctives (et pas seulement, par ex., à des complétives); c) assez simples pour pouvoir être utilisées dans chaque cas concret; 
d) de nature à conduire vers des résultats intuitivement acceptables.

7.2 Les parallèles structurales choisies, correspondant aux conditions posées, sont les suivantes:

Une expression de volonté est nécessairement prospective, elle vise le futur. Il n'est pas possible de commander et d'essayer d'influencer les faits ou les états dejà passés: la volonté $n^{\prime}$ est pas rétrospective.

Le premier critère est donc la question (1): l'action de la subordonnée peut-elle être antérieure à 1'action de la principale? Comme il n'est pas possible de commander "en arriere",

a) la réponse affirmative à la question (1) exclut l'emploi du subjonctif dans le sens de volonté.

7.3 La deuxieme parallèle structurale part de cette observation: l'une des qualités typiques du commentaire c'est de dire quelque chose sur ce qui se déroule, par conséquent, une action certaine, un état réel. Il en résulte un deuxième critère:

la question (2): l'action de la subordonnée est-elle présentée comme certaine, comme effectivement accomplie, réalisée?

Comme le commentaire présuppose la certitude de la réalisation de l'action qui est l'objet du commentaire,

(b) la réponse négative à la question (2) exclut l'emploi du subjonctif dans le sens de commentaire.

7.4 Une ultérieure observation est la suivante:

la qualité caractéristique du doute est de ne pas présenter une action comme certaine.

Par conséquent,

(c) la réponse affirmative à la question (2) exclut l'emploi du subjonctif dans le sens de doute.

Si l'on opère un croisement entre les domaines et les questions, on peut combiner

- la question (2) avec le domaine de la volonte,

- la question (1) avec le domaine du commentaire et du doute.

Dans le domaine de la volonté, quand on formule un ordre, par exemple, on $n^{\prime}$ est jamais sûr que le contenu de cet ordre sera effectivement réalisé, ce qui signifie que l'action exprimée 
par la subordonnée $\mathrm{n}^{\prime}$ est pas présentée comme certaine.

Par conséquent,

(d) la réponse affirmative à la question (2) exclut l'emp-

loi du subjonctif dans le sens de volonté.

Avec le commentaire, il est possible dire quelque chose sur ce qui s'était déjà passé.

Par conséquent,

(e) la réponse négative à la question (1) exclut l'emploi du subjonctif dans le sens de commentaire.

Un doute, on peut l'exprimer aussi à propos d'un fait antérieur.

Par consequent,

(f) une réponse négative à la question (1) exclut l'emploi du subjonctif dans le sens de doute.

7.5 Pour les deux parallèles structurales, on se servira des termes d'anteriorité (ANT) et de celui d'implication (IMPL). I1 s'agit, certes, de termes opérationnels qui ne sont pas à confondre avec les phénomenes linguistiques de l'antériorité et de la certitude supposée.

8. Par conséquent:

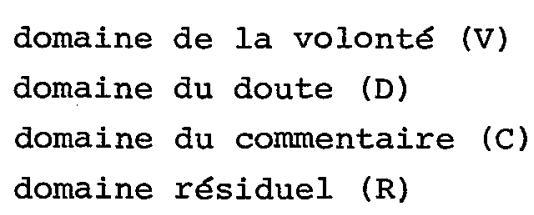

$\begin{array}{cc}\text { ANT } & \text { IMPL } \\ - & - \\ + & - \\ + & + \\ - & +\end{array}$

On nommera "le domaine résiduel" celui dans lequel on trouve des exemples, peu nombreux, d'ailleurs, où un élément lexical (une conjonction temporelle par ex.), definit nettement les relations temporelles dans ia phrase.

Exemples:

I. domaine de la volonté:

JE VEUX QU'IL AGISSE AINSI.

a. I'antériorité n'est pas possible:

b. l'action de la subordonnée n'est pas certaine

*JE VEUX QU'IL AIT AGI AINSI. la phrase cité ne suppose pas: IL AGIT AINSI 
II. domaine du doute:

a. 1'antériorité est possible:

b. I'action de la subordonnée $n^{\prime}$ est pas certaine;

III. domaine du commentaire:

a. l'antériorité de la subordonnée est possible:

b. I'action de la subordonnée est certaine:

IV. domaine résiduel:

a. l'antériorité de la subordonnée, à cause de la conjonction et de sa valeur lexicale, n'est pas possible;

b. l'action de la subordonnée est certaine;
JE DOUTE QU'IL AGISSE ATNSI. JE DOUIE QU'IL AIT AGI AINSI.

la phrase citée ne suppose pas: IL AGIT ATNSI.

JE SUIS CONIENT QU'IL AGISSE AINSI. JE SUIS CONIENT QU'IL AIT AGI AINSI. la phrase citée suppose: IL AGIT AINSI.

JE PARS AVANT QU'IL (NE) VIENNE ICI.

la phrase citée suppose: II VIENT ICI.

9. Ainsi, la conception de ces deux critères de l'antériorité et de l'implication de la certitude est-elle décrite, de même que la délimitation des domaines sémantiques. Mais, pour que ces deux instruments puissent devenir des outils de travail, il faut les élaborer, il faut formuler des procédés descriptifs ultérieurs dont deux sont fondamentaux.

9.1 Critère de l'antériorité:

on remplace la forme verbale de contemporanétié par celle de l'antériorité et ce test doit donner

a. une réponse positive, si une telle substitution a comme résultat une phrase acceptable;

b. une réponse négative, si la phrase n'est pas acceptable.

a. JE SUIS CONTENT QU'IL AGISSE AINSI.

b. JE SUIS CONTENT QU'IL AIT AGI AINSI.

b. JE VEUX QU'IL AGISSE AINSI. JE VEUX QU'IL AIT AGI AINSI.

9.2 Critère de l'implication:

On isole de la phrase en question la subordonnée en formant un segment autonome, une proposition; on elimine la conjonction et on se sert de la forme explicite du verbe. Le test de I'implication doit donner: 
a. une réponse positive si la subordonnée originelle, par le sens, correspond au segment isole;

b. une réponse négative si la subordonnée originelle, par son sens, ne correspond pas au segment isolé.

a. JE SUIS CONTENT QU'IL AGISSE AINSI.

Le segment isolé est: IL AGIT AINSI. L'action est présentée comme certaine, effective, comme elle l'est, d'ailleurs, dans la subordonnée. Par consequent, le segment et la subordonnée, par leur sens, concordent.

b. JE DOUTE QU'IL AGISSE AINSI.

Le segment II AGIT AINSI présente l'action comme certaine. Au contraire, la subordonnée originelle la présente comme non certaine. Donc, le segment et la subordonnee, par leur sens, ne concordent pas.

9.3 Les deux tests différent entre eux par leur nature:

a. le test de l'antériorité impose la modification du temps de la forme verbale dans ia subordonné;

b. le test de l'implication impose la modification du mode dans le verbe du segment.

10. Procédés descriptifs complémentaires.

Il est possible aussi de classifier les réponses aux deux tests par un simple critère intuitif sur

a. I'acceptabilité d'une proposition donné malgré la modification de la forme verbale dans la subordonné;

b. la concordance de sens entre l'action de la subordonnée et le segment isolé.

Dans la majeure partie des cas, 1'appreciation intuitive est suffisante; il y a quand même des champs limitrophes qui rendent 1'intuition incertaine. On propose pourtant des indicateurs linguistiques ulterieurs par lesquels on devrait obtenir des réponses aux tests plus claires et plus exactes. Ces indicateurs sont:

a. pour le critère de l'antériorité:

le test de I'adverbe adjoint à la subordonné:

b. pour le critère de l'implication de la certitude: 
le test d'un commentaire adjoint à la subordonnée;

le test de l'intercalation du sintagme LE FAIT QUE au lieu de la conjonction QUE (dans la complétive);

le test $d^{\prime}$ une réplique inverse adjointe.

10.1 Le test de 1'adverbe adjoint.

Le paradigme verbal de la contemporanéité est remplacé par le paradigme verbal de l'antériorité:

JE SUIS CONTENT QU!IL SOIT PRÉSENT A LA RÉUNION.

JE SUIS CONTENT QU'IL AIT ÉTÉ PRÉSENT A LA RÉUNION.

Dans le cas cite, au changement du paradigme verbal correspond aussi le changement de la perspective temporelle (l'antériorité). Pour un locuteur natif les deux phrases sont acceptables.

Toutefois, il existe des cas où la substitution du subjonctif présent ne provoque pas le changement de la perspective temporelle de la subordonnée: la forme verbale, le subjonctif passé, n'exprime pas une action preteritale, mais une action achevée:

JE VEUX QU'IL LE FINISSE VITE.

JE VEUX QU'IL L'AIT VITE FINI.

Or, si le subjonctif présent venait à être mécaniquement substitue par le subjonctif passé, on obtiendrait dans le cas cité une réponse positive au test de l'antériorité cela ne correspondrait pas au sens. A cause de cela, il faut distinguer les cas où le subjonctif passé exprime l'antériorité, de ceux où il exprime l'achèvement d'une action. Il est toujours possible d'entrevoir l'état réel à l'aide d'adverbes de temps appropriés que l'on ajoute à la subordonné, comme HIER, A L'ÉPOQUE, L'ANNÉE DERNIÈRE. Si l'emploi du subjonctif passé est compatible avec un tel adverbe, on peut alors en conclure que la subordonnée exprime 1'antériorité par rapport à la principale.

JE VEUX QU'IL L'AIT FINI DEMAIN.

*JE VEUX QU'IL L'AIT FINI HIER.

JE SUIS CONTENT QU'IL L'AIT FINI HIER.

10.2 Le test du commentaire adjoint.

Ce test est basé sur la supposition suivante: il est possi- 
ble de commenter l'action exprimée dans la subordonnée seulement, si cette action a été déjà presentée comme certaine dans la phrase originelle, si cette action est déjà là l'objet d'un commentaire. Autrement dit, si le commentaire adjoint à la subordonnée originelle rend une phrase francaise acceptable.

JE SUIS CONTENT QU'IL VIENNE; CELA ME REJOUIT MÊME.

JE VEUX QU'IL VIENNE, CELA ME REJOUIT MÊME. ${ }^{1}$

10.3 Le test du syntagme LE FAIT QUE (subordonnée complétive).

Ce test, employé seulement pour les subordonnées complétives se base sur la supposition que l'on peut remplacer la conjonction QUE par le syntagme LE FAIT QUE qui signifie "ce qui est vrai, réel" (Dict. Larousse, s.'v. 'fait'), seulement quand l'action de la subordonnée est presentée comme certaine.

Par conséquent, "LE FAIT QUE + l'action incertaine" n'est pas possible.

JE SUIS CONTENT QUE TU VIENNES.

JE SUIS CONTENT DU FAIT QUE TU VIENNES.

IL FAUT QU'ELLE PARTE.

*IL FAUT LE FAIT QU'ELLE PARTE.

Toutefois, 1'analyse des exemples dans le corpus montre que la présupposition qu'on trouve dans le dictionnaire n'est pas tout à fait valable. Le syntagme LE FAIT QUE est sémantiquement bien plus vide, comme l'a constaté déja Ronsjö 1966 ("De toute façon, LE FAIT semble avoir perdu aujourd'hui beaucoup de sa valeur sémantique").

A cause de cela, le syntagme est, jusqu'à un certain point, compatible aussi avec des completives de l'incertitude:

IL A PEUR QU'ELLE (NE) VIENNE.

IL A PEUR DU FAIT QU'ELLE (NE) VIENNE.

Le segment isolé, ELLE VIENT, ne correspond pas à la subordonnée citée et dont l'action est incertaine.

Le test, par conséquent, n'est pas un indicateur convenable.

1 Par son oeuvre Hot to Do Things with Worts, oxford 1962 , le philosophe J. Austin, en expliquant l'application d'un tel procede descriptif, a sensiblement influencé la description génerative des langues. 
10.4 Le test de réplique inverse adjointe

Ce test est basé sur la présupposition suivante: la subordonnee est compatible avec une annexe qui exprime une action opposé, seulement dans les cas où son action $n^{\prime}$ est pas présentée comme certaine. Il $n^{\prime}$ est donc pas possible de combiner une action certaine de la subordonnée avec 1'action inverse de la réplique.

IL VEUT QUE JE VIENNE, MAIS JE NE VIENDRAI PAS.

IL VEUT QUE JE NE VIENNE PAS, MAIS JE VIENDRAI QUAND-MÊME.

*JE SUIS CONTENT QUE TU VIENNES, MAIS TU NE VIENS PAS.

Dans ce travail on se servira du test de l'adverbe pour 1'antériorité et des tests du commentaire et de la réplique inverse pour 1'implication.

11. Illustration par des exemples du procédé descriptif.

11.1 Subordonnée complétive

IL A HONTE QUE SA MÈRE VIVE DE SES RENTES.

a. L'antériorité de la subordonnée est possible: IL A HONTE QUE SA MÈRE AIT VÉCU DE SES RENTES. En français, c'est une phrase acceptable. Il est possible aussi d'ajouter un adverbe convenable, AUTREFOIS par exemple.

b. L'action de la subordonnée est présentée comme certaine; le segment SA MÈRE VIT DE SES RENTES, qui présente l'action comme certaine, et la subordonnée concordent sémantiquement. L'idée intuitive est confirmée par la possibilité d'un commentaire adjoint: IL A HONTE QUE SA MÈRE VIVE DE SES RENTES, CE FAIT LUI EST TOUJOURS PÉNIBLE. D'après le schema (v. 8) cet exemple doit être classé dans le commentaire: ANT + , IMPL + .

IL DÉSIRE QUE TOUT SOIT EN ORDRE.

a. I'antériorité de la subordonnée n'est pas possible:

*IL DESIRE QUE TOUT AIT ÉTÉ EN ORDRE n'est pas une phrase française acceptable. Cela est confirmé par 1'incompatibilité de la subordonnée avec un adverbe de l'antériorité, par ex. HIER.

b. L'action de la subordonnée n'est pas présentée comme certaine: le segment TOUT EST EN ORDRE, qui présente l'action comme certaine, et la subordonnée ne concordent pas par leur sens. Ce jugement est confirme par l'incompatibilite de la phrase originelle avec le commentaire de la subordonnée: *IL DÉSIRE QUE 
TOUT SOIT EN ORDRE, MAIS EN VAIN, TOUT EST TOUJOURS EN DÉSORDRE.

La situation, ANT -, IMPL +, d'après notre schéma, classe cet exemple dans le domaine de la volonté.

IL CRAINT QU'ON (IN') APPRENNE LA VÉRITÉ.

a. L'antériorité de la subordonnée est possible: IL CRAINT QU'ON (N') AIT APPRIS LA VÉRITÉ est une phrase francaise acceptable. On peut aussi ajouter un adverbe d'antériorité convenable, HIER par exemple.

b. L'action de la subordonnée est présentée comme non-certaine: le segment ON APPREND(RA) LA VÉRITÉ, qui présente l'action comme certaine, et la subordonnée ne concordent donc pas par leur sens. Ce jugement est confirmé par l'incompatibilité de la phrase originelle avec le commentaire de la subordonnée: *IL CRAINT QU'ON (N') APRENNE LA VÉRITÉ, CELA LE BOULEVERSE. La situation, ANT +, IMPL -, d'après notre schéma, classe cet exemple dans le domaine du doute.

IL OBTIENT QU'ON LUI OBÉISSE.

a. L'antériorité n'est pas possible. *IL OBTIENT QU'ON LUI AIT OBEI n'est pas une phrase francaise acceptable. Une telle subordonnée n'est pas compatible avec un adverbe de l'anteriorite, par ex. HIER.

b. I'action de la subordonnée est présentée comme certaine: le segment ON IUI OBÉIT, qui présente l'action comme certaine, correspond sémantiquement à la subordonnée originelle. Ce jugement est confirmé par l'incompatibilite de la phrase originelle avec la réplique inverse de la subordonnée: *IL OBTIENT QU'ON LUI OBÉISSE, MAIS ON NE LUI OBEIT PAS.

Cette situation, ANT -, IMPL -, d'après notre schéma, classe cet exemple dans le domaine résiduel.

11.2 Subordonnée adverbiale

BIEN QU'IL FASSE DE SON MIEUX, SON DIRECTEUR N'EST PAS CONTENT.

a. L'antériorité de la subordonnée est possible: BIEN $Q U^{\prime} I L$ AIT FAIT DE SON MIEUX, SON DIRECTEUR N'EST PAS CONTENT est une phrase franqaise acceptable. Il est bien possible de lui ajouter une locution adverbiale de temps: JUSQU'A MAINTENANT. 
b. L'action de la subordonnée est présentée comme certaine: le segment IL FAIT DE SON MIEUX, qui présente 1'action comme certaine, et la subordonnée ne concordent pas par leur sens. Ce jugement est confirme par la compatibilité de la phrase avec le commentaire adjoint à la subordonnée: BIEN QU'IL FASSE DE SON MIEUX (CE QUE NOUS SAVONS TOUS), SON DIRECTEUR N'EST PAS CONTENT.

La situation, ANT +, IMPL +, classe, d'après notre schéma, cet exemple dans le domaine du commentaire.

IL FAIT TOUT POUR QU'ELLE SOIT HEUREUSE.

a. L'antériorité de la subordonnée n'est pas possible: *IL FAIT TOUT POUR QU'ELLE AIT ÉTÉ HEUREUSE n'est pas une phrase francaise acceptable.

b. L'action de la subordonnée est présentée comme non-certaine: le segment ELLE EST HEUREUSE, qui présente l'action comme certaine, et la subordonnée originelle, par leur sens ne concordent pas. Ce jugement est confirmé par la possibilité d'ajouter à la subordonnée la réplique inverse: IL FAIT TOUT POUR QU'ELLE SOIT HEUREUSE, ET POURTANT ELLE N'EST PAS HEUREUSE.

La situation, ANT -, IMPL -, classe cet exemple dans le domaine de la volonté.

IL EST TRÈS AIMABLE POURVU QU'ON FASSE BIEN SON DEVOIR.

a. L'antériorité de la subordonnée est possible: II EST TRÈS AIMABLE POURVU QU'ON AIT BIEN FAIT SON DEVOIR est une phrase francaise acceptable. Il est aussi possible d'ajouter un adverbe d'antériorité, AUPARAVANT par exemple.

b. L'action de la subordonnée est présentée comme certaine: le segment ON FAIT BIEN SON DEVOIR, qui présente l'action comme certaine, et la subordonnée, ne concordent pas sémantiquement. Ce jugement est confirmé par la compatibilité de la phrase originelle avec la réplique inverse de la subordonněe: IL EST TRÈS AIMABLE POURVU QU'ON FASSE BIEN SON DEVOIR, MAIS SI ON NE LE FAIT PAS BIEN, ALORS IL N'EST PAS AIMABLE.

La situation, ANT +, IMPL -, classe cet exemple dans le domaine du doute.

JE VAIS CUEILLIR QUELQUES FLEURS AVANT QUE TU (NE) VIENNES ICI. 
a. L'antériorité de la subordonnée, à cause du sens de la conjonction AVANT QUE, n'est pas possible. L'analyse logique témoigne que l'action de la subordonnée dans ce cas est toujours postérieure à l'action de la principale. Il serait donc illogique de vouloir ajouter des adverbes de temps.

b. L'action de la subordonnée présentée comme certaine. Le segment TU VIENDRAS ICI, qui présente l'action comme certaine, et la subordonnée concordent par leur sens. Ce jugement est confirmé par la possibilité d'ajouter un commentaire à la subordonnée: PARCE QUE TA VISITE ME FAIT PLAISIR. En même temps l'incompatibilité de la phrase originelle avec la réplieu que inverse de la subordonne le confirme: *JE VAIS CUEILLIR QUELQUES FLEURS AVANT QUE TU (NE) VIENNES ICI, MAIS TU NE VIENS PAS ICI.

La situation, ANT -, IMPL +, classe cet exemple dans le domaine résiduel.

11.3 Subordonnée relative

C'EST IE SEUL ARTICLE QUE NOUS AYONS EN MAGASIN.

a. L'antériorité est possible: C'EST LE SEUL ARTICLE QUE NOUS AYONS EU EN MAGASIN est une phrase francaise acceptable. Il est possible aussi de lui adjoindre une locution adverbiale d'antériorité, per ex. A L'ÉPOQUE.

b. L'action de la subordonnée est présentée comme certaine: le segment NOUS AVONS UN SEUL ARTICLE EN MAGASIN, qui présente l'action comme certaine, et la subordonnée originelle concordent par leur sens. Ce jugement est confirmé par la compatibilité de la phrase avec le commentaire adjoint d̀ la subordonnée: C'EST LE SEUL ARTICLE QUE NOUS AYONS EN MAGASIN, CE QUI PROUVE LES DIFFICULTÉS D'APPROVISIONNEMENT.

La situation, ANT +, IMPL +, classe, d'après notre schéma, cet exemple dans le domaine du commentaire.

ILS SONT DECIDÉS A ADOPTER UN CHIEN QUT LEUR TIENNE COMPAGNIE.

a. L'antériorité de la subordonnée n'est pas possible: *ILS SONT DÉCIDÉS A ADOPTER UN CHIEN QUI LEUR AIT TENU COMPAGNIE $n^{\prime}$ est pas une phrase francaise acceptable. L'éventuelle addition d'une locution adverbiale d'antériorité, par ex. JUSQU'A MAINTENANT, ne change pas la situation. 
b. L'action de la subordonnée n'est pas présentée comme certaine: le segment UN CHIEN LEUR TIENT COMPAGNIE, qui présente l'action comme certaine, et la subordonnée originelle ne concordent pas par leur sens. Ce jugement est confirme par l'incompatibilité de la subordonnée originelle avec le commentaire: *ILS SONT DÉCIDÉS A ADOPTER UN CHIEN QUI LEUR TIENNE COMPAGNIE, ET ILS L'AIMENT BIEN.

La situation, ANT -, IMPL -, classe, d'après notre schéma, cet exemple dans le domaine de la volonté.

CONNAISSEZ-VOUS UN CHEVAL QUI COURE CETTE DISTANCE PLUS VITE?

a. L'antériorité de la subordonnée est possible: CoNNAIsSEZ-VOUS UN CHEVAL QUI AIT COURU CETTE DISTANCE PLUS VITE? est une phrase francaise acceptable. Il est bien possible aussi de lui ajouter une locution adverbiale d'antériorité convenable, par ex. L'ANNÉE DERNIÈRE.

b. I'action de la subordonnée n'est pas présentée comme certaine: le segment UN CHEVAL COURT CETTE DISTANCE PLUS VITE, qui présente $I^{\prime}$ action comme certaine, et la subordonnée originelle, ne concordent pas quant au sens. Ce jugement est confirmé par l'incompatibilité de la phrase originelle avec le commentaire adjoint de la subordonnee: *CONNAISSEZ-VOUS UN CHEVAL QUI COURE CETTE DISTANCE PLUS VITE? CELA ME SURPREND BIEN.

La situation, ANT +, IMPL -, classe cet exemple, d'après notre schéma, dans le domaine du doute.

12. Le subjonctif apparâ̂t aussi, bien que peu fréquemment, dans les indépendantes ou apparemment independantes (dans notre corpus, c'est à peu près $10 \%$ d'exemples). L'analyse de l'emploi du subjonctif montre qu'il s'agit de deux situations facilement definissables:

a. I'impératif de la 3.ème personne;

b. 1'emploi du plus-que-parfait du subjonctif à valeur hypothetique.

On est en général d'accord pour voir dans l'impératif QU'IL VIENNE la structure profonde JE VEUX QU'IL VIENNE; et de voir 
dans l'emploi hypothétique UN BATAILLON NE L'EÛT PAS FAIT RECULER la structure ON DIT (présent) QU'UN BATAILLON NE L'EÛT PAS FAIT RECULER.

Les propositions de ces deux catégories se classent donc respectivement dans le domaine de la volonté et du doute.

13. Il faut préciser que les critères de l'implication et de l'antériorité diffèrent entre eux quant à certains traits caractéristiques.

13.1 On verifie l'implication en faisant en sorte que la proposition originelle ne subisse aucun changement. Puis on compare à cette proposition le segment qui doit rester le plus fidèle possible à la subordonnée originelle. On ne doit admettre qu'une commutation minimale: disparition de la conjonction, modification de la forme verbale. Le segment contient tous les élements linguistiques qui apparaissent dans la subordonnée. Le test de l'implication cherche à vérifier si le sens du segment correspond au sens de la subordonnée ou pas.

13.2 On vérifie l'antériorité en modifiant la subordonnée originelle: les formes verbales de la contemporanété sont remplacées par celles de l'antériorité. La question de savoir si la phrase, ayant subi des modifications, est encore une phrase francaise acceptable, se pose alors.

De ce point de vue, les "phrases-modèle" ne se présentent pas comme trop complexes, et pour cause: elles ne contiennent $\mathrm{ni}$ des éléments lexicaux $\mathrm{ni}$ un contexte (la logique du discours) qui peuvent toujours s'opposer à de telles modifications. Prenons par exemple une phrase très simple comme JE DOUTE QU'IL PUISSE VENIR DEMAIN. EIle n'admet pas 1'anteriorité *JE DOUTE QU'IL AIT PU VENIR DEMAIN. Par contre, la proposition sans adverbe ne présente aucun obstacle pour exprimer l'antériorite: JE DOUTE QU'IL AIT PU VENIR.

14. La vue theorique qu'on vient de présenter se fonde sur les analyses et les évaluations des exemples que donnent les grammaires, les manuels et les oeuvres spécialisées, controlés (et méités) par les lecteurs de francais a Ljubljana. Mais, ce sont avant tout les passages dans le corpus choisi 
qui ont eu un poids décisif. En effet, le materiel du corpus, des oeuvres littéraires, présente souvent des difficultés pour l'analyse, parce que les phrases contiennent souvent des structures complexes, aussi complexes que les facteurs qui imposent le choix du mode verbal.

Certes, I'avantage des phrases-modèle est justement dans leur relative clarté, parce que - etant des modèles - elles sont construites simplement, avec l'intention precise d'eclairer un problème, ou encore, un seul secteur de l'emploi du paradigme verbal. Par conséquent, ces phrases ne sont pas conditionnées par toute une serie de facteurs lexicaux, syntaxiques et pragmatiques, par le contexte. Cette indépendance provoque en même temps des doubles sens, ce qui est plus rare dans un contexte litteraire. La phrase-modele C'EST MIEUX QUE MARX NE SOIT PLUS A IA MAISON peut être différemment interprétée et, effectivement, elle 1'a été par des locuteurs natifs. Ce Marx peut ne pas être chez lui et nous sommes donc dans le domaine du commentaire, ou bien Marx peut se trouver toujours à la maison et on exprime alors le désir de le voir s'en aller; dans ce cas nous sommes dans le domaine de la volonté. Le passage littéraire (La Chronique de Vissoko, 166) par contre n'admet pas une double interprétation: Marx n'est plus chez lui, la phrase n'est donc interprétable que comme commentaire d'une action certaine..

15. Le système présenté se fonde sur les matériaux qu'offrent quatre oeuvres litteraires, deux francaises et deux slovènes, et leurs respectives traductions; ce sont:

Gustave Flaubert, Madame Bovary - Gospa Bovaryjeva (MB)

Gustave Flaubert, L'Éducation sentimentale - Vzgoja srca (ES)

Ivan Tavčar, Visoška kronika - La Chronique de Vissoko (VK)

Ciril Kosmač, Balada o trobenti in oblaku - La ballade de la trompette et du nuage (BT)

Dans ce corpus, les formes du subjonctif (quelque onze cents cas) apparaissent avec une fréquence moyenne d'une forme subjonctive, clairement constatable, à 1,2 page.

Ces matériaux ont été soumis à l'analyse, fondée sur les prémisses théoriques. Certes, la structuration de la phrase est 
parfois trés complexe ce qui rend difficile d'isoler les qualités structurales d'une situation donnée dans laquelle le subjonctif apparâ̂t lié à un verbe donnée. On a constamment à faire d'une part à mécanismes syntaxiques et lexicaux et, d'autre part, à $l^{\prime}$ influence du contexte, voire du cadre plus ample.

15.1 Il faut se rendre compte des dangers, même des simples erreurs typographiques; on trouve, dans I'Éducation sentimentale, p. 297 MALGRÉ LA LÉGISLATION LA PLUS HUMAINE QUI fut JAMAIS, LE SPECTRE DE 93 REPARUT.

Il faut éliminer les phraséologuèmes qui ne sont plus analysables: TANT SOIT PEU, DIEU SOIT LOUÉ, GRAND BIEN LUI FASSE, N'EN DÉPLAISE A QUELQU'UN, VIVE + substantif.

16. La permutation du subjonctif avec l'indicatif. Si l'on applique les études faites sur la possibilité de la permutation des deux modes verbaux (cfr. l'étude de w. Rothe) à notre classification, on peut constater que dans le domaine

a. de la volonté, le subjonctif n'est pas échangeable avec l'indicatif, ni dans la langue parlée ni dans la langue littéraire;

b. du commentaire, le subjonctif, dans l'emploi non marque, n'est pas echangeable avec I'indicatif, sauf dans la langue parlée, non formelle;

c. du doute, les deux modes verbaux sont interchangeables; les cas sont relativement fréquents, dans la langue littéraire et non-littéraire.

17. Exemples illustratifs.

17. I La subordonnée complétive.

1. MB 256 SEULEMENT, ELLE REGRETTAIT QUE SON MARI N'EÛT PAS RECU LES SECOURS DE LA RELIGION.

IMPL: SON MARI N'AVAIT PAS RECU LES SECOURS DE LA RELIGION. Le sens du segment concorde avec celui de la subordonnée - tous les deux présentent l'action comme certaine.

ANT: L'antériorité de l'action de la subordonnée est exprimée dans la subordonnée meme.

La situation, IMPL +, ANT +, classe l'exemple 
dans le domaine du commentaire.

2. MB 30 LA MARIÉE AVAIT SUPPLIÉ SON PÈRE QU'ON LUI EPARGNÂT LES PLAISANTERIES D'USAGE/.

IMPL: ON LUI EPARGNA LES PLAISANTERIES D'USAGE.

Le segment et la subordonnée ne concorde pas par leur sens; le segment présente l'action comme certaine, tandis que la subordonnée la présente comme non-certaine.

ANT: Le verbe SUPPLIER (comme le démontre la phrase-modèle correspondante) n'admet pas une subordonnée d'antériorité.

La situation, IMPL +, ANT -, classe cet exemple dans le domaine de la volonté.

3. MB 249 LÉON AVAIT PEUR QU'ELLE NE RENTRÂT DANS L'ÉGLISE. IMPL: ELLE RENTRA DANS L'ÉGLISE.

Le sens du segment, qui montre l'action comme certaine, ne concorde pas avec le sens de la subordonnée, laquelle, au contraire, montre l'action comme non-certaine.

4. MB 8 A DOUZE ANS, SA MÈRE OBTINT QUE L'ON COMMENÇÂT SES ÉTUDES.

IMPL: (A DOUZE ANS) L'ON COMMENGA SES ÉTUDES.

Le segment et la subordonnée concordent sémantiquement. Tous les deux présentent 1'action comme certaine.

ANT: La phrase-modèle correspondante montre qu'une telle situation avec le subjonctif d'une manière générale n'est pas compatible avec la subordonnée qui exprime l'antériorité.

La situation, IMPL +, ANT -, classe l'exemple dans le domaine du but atteint.

17.2 La subordonnée adverbiale.

1. MB 259 C'EST UN BRAVE GARCON QUOIQUE NOUS AYONS EU ENSEMBLE DES DIFFICULTÉS.

IMPL: NOUS AVONS EU ENSEMBLE DES DIFFICULTÉS. Le sens du segment concorde avec celui de la subordonnée - tous les deux présentent I'action comme certaine. 
ANT: Dans la subordonnée est exprimée l'antériorite; la compatibilité supposée de la phrase-modèle correspondante avec l'antériorité est donc confirmée par la subordonnée meme.

La situation, IMPL +, ANT +, classe cet exemple dans le domaine du commentaire.

2. MB 115 JE LA DONNERAI AU CURÉ, POUR QU'IL ME DISE DES MESSES.

IMPL: IL ME DIRA DES MESSES.

La segment présente l'action comme certaine, la subordonnée comme probable, mais pas certaine. Le segment et la subordonnée ne concordent donc pas par leur sens.

ANT: L'antériorité n'est pas possible.

La situation, IMPL -, ANT -, classe cet exemple dans le domaine de la volonté.

3. MB 243 MAIS CHARLES REPONDIT QU'IIS S'EN ALLAIENT DÈS LENDEMAIN. "A MOINS, AJOUTA-T-IL EN SE TOURNANT VERS SA FEMME, QUE TU NE VEUILLES RESTER SEULE, PETIT CHAT?"

IMPL: TU VEUX RESTER SEULE.

Le segment présente l'action comme certaine, la subordonnée la présente comme non-certaine. Ils ne concordent donc pas par leur sens.

ANT: La phrase-modèle correspondante montre que, en général, cette situation est compatible avec 1 'antériorité.

La situation, IMPL -, ANT +, classe cet exemple dans le domaine du doute.

4. MB 36 AVANT QU'ELLE SE MARIÂT, ELLE AVATT CRU AVOIR DE L'AMOUR. .

IMPL: ELLE SE MARIA.

ANT: A cause de la conjonction temporelle, l'antériorité $n^{\prime}$ est pas possible.

La situation, IMPL +, ANT -, classe cet exemple dans le commentaire, limité par la conjonction temporelle. 
17.3 La subordonnée relative.

1. MB 351 C'EST LE PREMIER QUI AIT FAIT VENIR DANS LA SEINE-INFÉRIEURE DU CHO-CA ET DE LA REVALENTIA.

IMPL: LUI PREMIER A FAIT VENIR DANS LA SEINE-INFÉRIEURE.. Le segment et la subordonnée montrent l'action comme certaine. Par conséquent, ils concordent par leur sens.

ANT: L'antériorité est exprimee dans la subordonnée même.

La situation, IMPL +, ANT +, classe cet exemple dans le commentaire.

2. MB 314 PUIS IL FAUDRAIT INVENTER UNE HISTOIRE QUI EXPLIQUÂT LES CHOSES A BOVARY.

IMPL: UNE HISTOIRE EXPLIQUA LES CHOSES A BOVARY.

Le segment montre $I^{\prime}$ action comme certaine; la subordonnée montre $I^{\prime}$ action comme non-certaine. Par consequent, ils ne concordent pas par leur sens.

ANT: L'antériorité n'est pas possible. IL FAUT INVENTER concorde par sa valeur sémantique avec le verbe CHERCHER.

La situation, IMPL -, ANT -, classe cet exemple dans le domaine de la volonté.

17.4 La proposition indépendante principale.

1. MB 334 QU'ON NE ME DISE RIEN, J'AURAI DE LA FORCE...

IMPL: On part de la structure profonde JE VEUX QU'ON NE ME DISE RIEN.

Le segment isolé est ON NE ME DIT RIEN qui présente l'action comme certaine, ce qui n'est pas le cas de la proposition (apparemment) principale. Ils ne concordent donc pas par leur sens.

ANT: L'antériorité n'est pas possible.

La situation, IMPL -, ANT -, classe l'exemple dans le domaine de la volonté.

2. MB 326 L'APPARITION D'UN DIEU N'EÛT PAS CAUSÉ PLUS D'ÉMOI. IMPL: On part de la structure profonde (ON DIT QUE) 
L'APPARITION D'UN DIEU N'EÛT PAS CAUSÉ PLUS D'ÉMOI. Le segment isolé est L'APPARITION D'UN DIEU $N^{\prime} A$ PAS CAUSÉ PLUS D'ÉMOI.

Le segment isole est L'APPARITION D'UN DIEU N'A PAS CAUSÉ... L'action est présentée par le segment comme certaine; elle est présentée comme non-certaine (non-rélle) par la subordonnée. Ils ne concordent donc pas par leur sens.

ANT: La structure profonde suppose I'existence du verbe DIRE, au présent; la subordonnée, dans la période ainsi concue, exprime l'antériorité.

La situation, IMPL -, ANT +, classe I'exemple dans le domaine du doute.

18. LES PHRASES AVEC LE SUBJONCTIF DANS NOTRE CORPUS. IA LISTTE DES ÉLÉMENTS DISTINCTIFS D'APRÈS LES SITUATIONS ET D'APRÈS LE CLASSEMENT SÉMANTIQUE.

Les résultats de 1'analyse sémantique seront présentés à part pour les oeuvres françaises originales et pour les oeuvres traduites en français. Les exemples seront classés d'après les types de la proposition. Chaque element distinctif sera précédé, entre parenthèse, de la classification qu'a ou qu'aurait la phrase-modèle. Dans ce classement les emplois obligatoires du subjonctif (plus-que-parfait du subjonctif à valeur hypothétique) n'apparaîtront pas.

On utilisera les lettres $v, d, c, c_{t}$ pour les domaines de la volonté, du doute, du commentaire, du commentaire avec une précision temporelle. 
PROPOSITION COMPLÉTIVE

(v/d) admettre

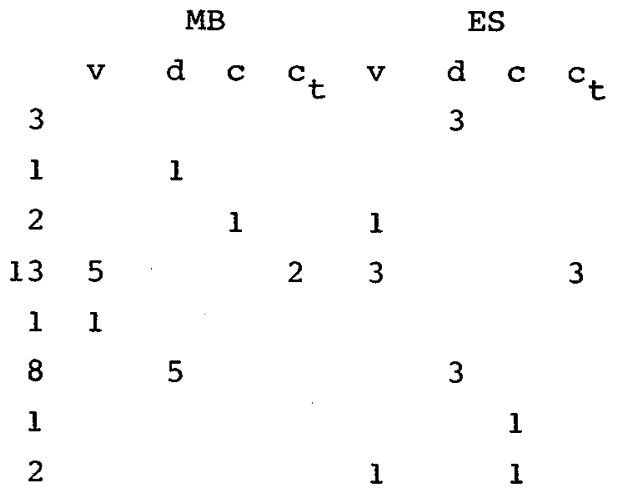

(d) affirmer

(c) aimer

(v) attendre

(v) avertir

(d) avoir peur

(v) il n'est pas besoin

( $v / c)$ être bien le moins

(v) commander

(c) comprendre

(d) craindre

(d) crainte

(d) croire

(v) demander

(v) désirer

(d) douter

(c) ne pas se douter

(v) empêcher

(v) envie

(c) s'étonner

(c) étonné

(v) exiger

(c) fâché

11

5

6

I

I

11

31

11

3

2

21

1

3

2

4

1

5

(v) faire

(v) il faut

(d) s'imaginer

(c) s'indigner

$(\mathrm{v} / \mathrm{c})$ inutile

(v) laisser

$\left(c_{t}\right)$ obtenir

(v) s'opposer à ce

(v) ordonner

(d) penser

11

2

1

21

3

31

6

2

271

2

$\begin{array}{lll}1 & 1 & 1 \\ & 2 & \end{array}$

1

1

3

1

1

1

11

1

l 1

2

2

1

1 


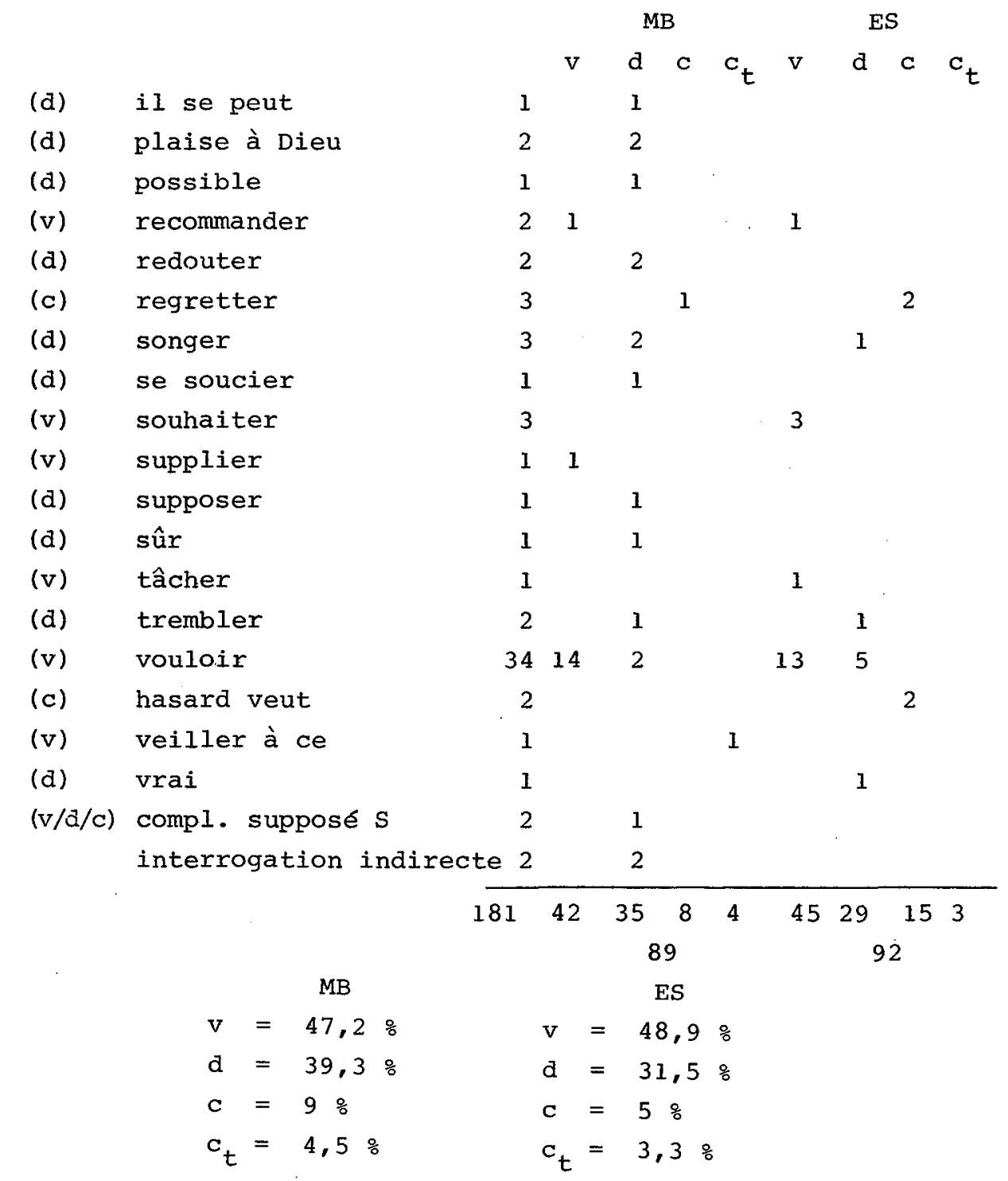

PROPOSITION ADVERBIALE

(v) afin que

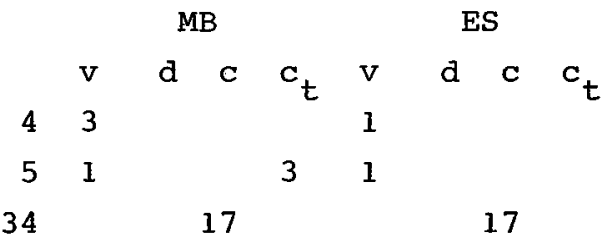


comme

comme si

(d) ne fût-ce que

(ct) jusqu'a ce que

(d) à moins que

(v) de peur que

(v) pour que

(d) trop...pour que

(d) pour peu que

(d) pourvu que

(d) ce n'est pas que

(v) que "pour que"

(d) que "si"

(d) que.. ou que

(d) quel que

(d) quelque.. que

(c) quoique

(d) quoi que si

(d) si.. et que inversion hypothétigue (d) non pas que

\begin{tabular}{|c|c|c|c|c|c|c|c|}
\hline & & & & & & & \\
\hline & $\mathrm{v}$ & $d$ & C & $c_{t}$ & $\mathrm{v}$ & $d$ & C \\
\hline 5 & & 4 & & & & 1 & \\
\hline 45 & & 25 & & & & 21 & \\
\hline
\end{tabular}

22

1

2

22

$29 \quad 13$

1

11

32

11

1

11

11

22

32

11

$25 \quad 20$

$\begin{array}{lll}7 & 2 & 8\end{array}$

118

11

(d) si... que

(d) soit que.. ou que aussi adj. que si

41

10

2

plus adj. que si

plus adj. que

autant de nom que si

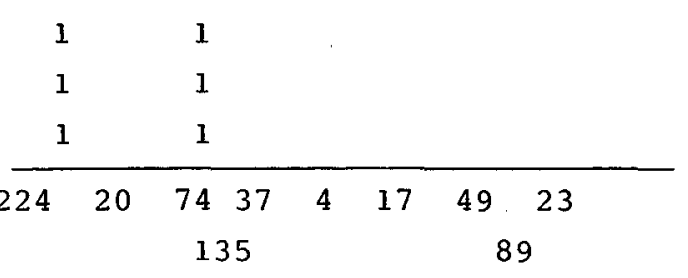

ES

$\begin{array}{rlrl}\mathrm{v} & =14,8 \% & \mathrm{v} & =19,1 \% \\ \mathrm{~d} & =54,8 \% & \mathrm{~d}=55,1 \% \\ \mathrm{c} & =27,4 \% & \mathrm{c}=25,8 \% \\ \mathrm{ct} & =3 \% & & \end{array}$


PROPOSITION RELATIVE

(c) le plus

(c) le seul

(c) le premier

(d) personne

(d) pas un

(d) rien

(v) quelque chose

(v) quelqu'un

(v/d) une chose

une, quelque chose

\begin{tabular}{|c|c|c|c|c|c|c|c|c|}
\hline & \multicolumn{4}{|c|}{ MB } & \multicolumn{4}{|c|}{ ES } \\
\hline & $\mathrm{v}$ & d & $\mathrm{C}$ & $c_{t}$ & $\mathrm{v}$ & $\mathrm{d}$ & C & $c_{t}$ \\
\hline 5 & & & 3 & & & & 2 & \\
\hline 8 & & 1 & 3 & & & & 4 & \\
\hline 1 & & & 1 & & & & & \\
\hline 4 & & $I$ & & & 1 & 2 & & \\
\hline 4 & & 1 & & & & 3 & & \\
\hline 1 & 1 & & & & & & & \\
\hline 1 & & & & & 1 & & & \\
\hline 1 & & & & & 1 & & & \\
\hline 9 & 7 & & & & 2 & & & \\
\hline 10 & & 8 & & & & 2 & & \\
\hline 44 & 8 & 11 & 7 & & 5 & 7 & 6 & \\
\hline & & & & & & & 8 & \\
\hline
\end{tabular}

ES

$\mathrm{v}=27,8 \%$

$\mathrm{d}=38,9 \%$

$c=33,3 \%$

PROPOSITION INDÉPENDANTE/PRINCIPALE

(v) l'impératif-optatif avec que
optatif pourvu que

(v) optatif pourvu que<smiles>[113CH2]</smiles>

(v) optatif sans que subj. plpf.

$\begin{array}{rrr}19 & 7 & 12 \\ 3 & 1 & 2 \\ 1 & 1 & \end{array}$

$v \quad d \quad c \cdot c_{t} \cdot v \quad d \quad c \quad c_{t}$ 
19. TABLEAU DES TYPES DE PROPOSITIONS D'APRÈS LES DOMAINES SÉMANTIQUES

TYPE DE PROPOSITION

subordonnée complétive

\begin{tabular}{rrrlrrrl}
\multicolumn{1}{c}{ MB } & \multicolumn{1}{c}{$E S$} \\
$\mathrm{v}$ & $\mathrm{d}$ & $\mathrm{c}$ & $\mathrm{c}_{\mathrm{t}}$ & $\mathrm{v}$ & $\mathrm{d}$ & $\mathrm{c}$ & $\mathrm{c}_{\mathrm{t}}$ \\
42 & 35 & 8 & 4 & 45 & 29 & 15 & 3 \\
20 & 74 & 37 & 4 & 17 & 49 & 23 & - \\
8 & 11 & 7 & - & 5 & 7 & 6 & - \\
9 & 27 & - & - & 14 & 12 & - & - \\
\hline 79 & 147 & 52 & 8 & 81 & 97 & 44 & 3 \\
& 286 & & & 225 &
\end{tabular}

subordonnée adverbiale

subordonnee relative

proposition principale

ES

$\begin{aligned} & \text { MB } \\ \mathrm{v} & =28 \% \\ \mathrm{~d} & =51 \% \\ \mathrm{c} & =18 \% \\ \mathrm{c}_{\mathrm{t}} & =3 \%\end{aligned}$

$\mathrm{v}=36 \%$

$\mathrm{d}=43 \%$

$\mathrm{c}=20 \%$

$c_{t}=1 \%$

DOMAINE SÉMANTIQUE

\begin{tabular}{lrr} 
& MB & ES \\
domaine de la volonte & $28 \%$ & $36 \%$ \\
domaine du doute & $51 \%$ & $43 \%$ \\
domaine du commentaire & $18 \%$ & $20 \%$ \\
domaine du but atteint-commentaire & $3 \%$ & $1 \%$ \\
\cline { 2 - 3 } & $100 \%$ & $100 \%$
\end{tabular}

20. LA CHRONIQUE DE VISSOKO ET LA BALLADE DE LA TROMPETTE ET DU NUAGE

PROPOSITION COMPLÉTIVE

(v/c) accepter

(d) affirmer

(c) aimer

$\mathrm{VK}$ BT

(d) il arrive

\begin{tabular}{llccccccc} 
& \multicolumn{3}{c}{$\mathrm{VK}$} & \multicolumn{4}{c}{$\mathrm{BT}$} \\
& $\mathrm{v}$ & $\mathrm{d}$ & $\mathrm{c}$ & $\mathrm{c}_{\mathrm{t}}$ & $\mathrm{v}$ & $\mathrm{d}$ & $\mathrm{c}$ & $\mathrm{c}_{\mathrm{t}}$ \\
1 & 1 & & & & & & & \\
1 & & & & & & 1 & & \\
3 & 2 & & & & 1 & & & \\
3 & & 2 & & & & & &
\end{tabular}




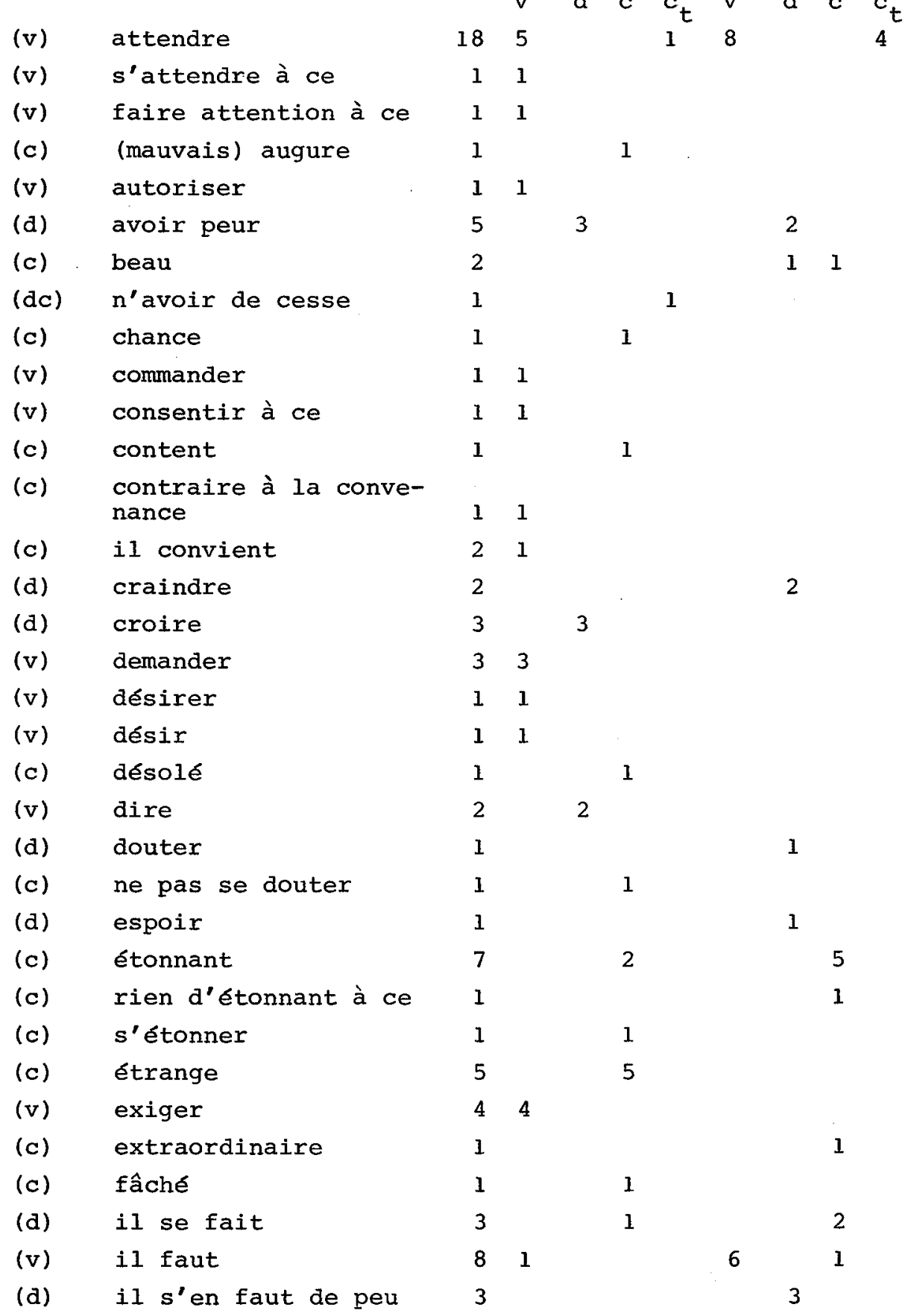









\begin{tabular}{|c|c|c|c|c|c|c|c|c|c|c|c|c|}
\hline \multirow[b]{3}{*}{ (v) } & \multirow{3}{*}{\multicolumn{3}{|c|}{ vouloir }} & & \multicolumn{4}{|c|}{ VK } & \multicolumn{4}{|c|}{$\mathrm{BT}$} \\
\hline & & & & & $\mathrm{v}$ & d & c & $c_{t}$ & $\mathrm{v}$ & $d$ & c & $c_{t}$ \\
\hline & & & & 10 & 6 & & & & 3 & 1 & & \\
\hline (c) & chance & veut & & 1 & & & 1 & & & & & \\
\hline \multirow[t]{8}{*}{$(v / d / c)$} & comp 1 . & supposé & $\mathrm{s}$ & 3 & & & 3 & & & & & \\
\hline & & & & 175 & 52 & 19 & 32 & 2 & 22 & 24 & 20 & 4 \\
\hline & & & & & & & 05 & & & 70 & & \\
\hline & & VK & & & & $\mathrm{BT}$ & & & & & & \\
\hline & $v$ & $=49,5$ & & & $\mathrm{v}$ & $=$ & 31,4 & 양 & & & & \\
\hline & $\mathrm{d}$ & $8 \%$ & & & $d$ & $=$ & 34,3 & $\%$ & & & & \\
\hline & c & $=30,5$ & & & c & $=$ & 28,6 & $\%$ & & & & \\
\hline & $c_{t}$ & $2 \%$ & & & $c_{t}$ & & 5,7 & 웅 & & & & \\
\hline
\end{tabular}

PROPOSITION ADVERBIALE

(v) afin que

$\left(c_{t}\right)$ après que

(d) autant que

$\left(c_{t}\right)$ avant que

(c) bien que

comme

comme si

(c) encore que

(v) de (telle) facon que

(d) fût-ce

$\left(c_{t}\right)$ jusqu'à ce que

(d) à moins que

(d) où que

(v) pour que

(v/c) assez.. pour que

(v) pour ne pas que

(v) que "pour que"

(d) que.. ou

(d) quel que

(d) quelque nom que

\begin{tabular}{|c|c|c|c|c|c|c|c|c|}
\hline & \multicolumn{4}{|c|}{ VK } & \multicolumn{4}{|c|}{$\mathrm{BT}$} \\
\hline & $\mathrm{v}$ & $d$ & c & $c_{t}$ & $v$ & $\mathrm{~d}$ & c & $c_{t}$ \\
\hline 5 & 5 & & & & & & & \\
\hline 6 & & & 4 & & & & & 2 \\
\hline 3 & & 3 & & & & & & \\
\hline 13 & 5 & & 4 & & 4 & & & \\
\hline 66 & & & 36 & & & & 30 & \\
\hline 1 & & 1 & & & & & & \\
\hline 2 & & & & & & 2 & & \\
\hline 6 & & & & & & & 6 & \\
\hline 2 & 2 & & & & & & & \\
\hline 1 & & 1 & & & & & & \\
\hline 14 & & & 11 & & & & & 3 \\
\hline 1 & & 1 & & & & & & \\
\hline 1 & & 1 & & & & & & \\
\hline 51 & 43 & & & 2 & 6 & & & \\
\hline 3 & 1 & & 1 & & & & 1 & \\
\hline 1 & & & & & 1 & & & \\
\hline 8 & 2 & & & & 6 & & & \\
\hline 4 & & 4 & & & & & & \\
\hline 1 & & 1 & & & & & & \\
\hline 5 & & 5 & & & & & & \\
\hline
\end{tabular}


(c) quoique

(d) quoi que

(d) sans que

(d) si adj. que

(d) soit que.. ou que

\begin{tabular}{|c|c|c|c|c|c|c|c|c|}
\hline & & & $7 \mathrm{~K}$ & & & B & & \\
\hline & $\mathrm{v}$ & $a$ & $c$ & $c_{t}$ & $\mathrm{v}$ & $d$ & $\mathrm{c}$ & $c_{t}$ \\
\hline 2 & & & & & & & 2 & \\
\hline 3 & & 2 & & & & 1 & & \\
\hline 3 & & 1 & & & & 2 & & \\
\hline 2 & & 2 & & & & & & \\
\hline 6 & & 6 & & & & & & \\
\hline 210 & 58 & 28 & 37 & 21 & 17 & 5 & 39 & 5 \\
\hline & & & 144 & & & & 6 & \\
\hline
\end{tabular}

$$
\begin{aligned}
& \text { VK } \\
& \mathrm{v}=40,3 \% \\
& \mathrm{~d}=19,4 \% \\
& \mathrm{c}=25,7 \% \\
& \mathrm{c}_{t}=14,6 \%
\end{aligned}
$$

BT

$$
\begin{array}{r}
\mathrm{v}=25,8 \% \\
\mathrm{~d}=7,6 \% \\
\mathrm{~d}=59,8 \% \\
\mathrm{c}_{t}=8,6 \%
\end{array}
$$

PROPOSITION RELATIVE

(c) le plus

(c) le pire

(c) peu de nom

(d) personne

(d) rien

(v) quelque chose

(c) expression affective

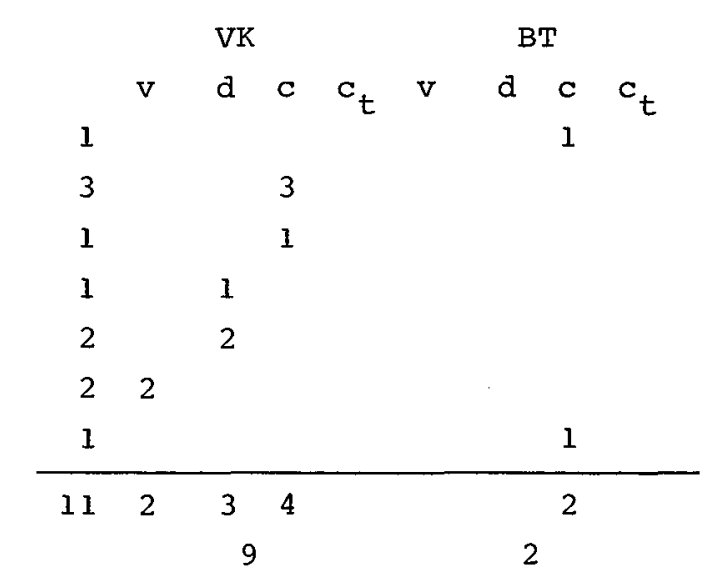

BT

$C=100 \%$ 
PROPOSITION INDÉPENDANTE/PRINCIPALE

(v) imperatif-optatif avec

que $26 \quad 24 \quad 2$

(v) optatif pourvu que $\quad 3 \quad 1 \quad 2$

(v) optatif sans que 33

(d) plpf. du subj.

\begin{tabular}{lll}
2 & 2 \\
\hline 3428 & 4 & 2
\end{tabular}

$\mathrm{VK}=100 \%$
$\mathrm{v}=66,7 \%$
$\mathrm{~d}=33,3 \%$

LES TYPES DE SUBORDONNÉES D'APRÈS LES DOMAINES SÉMANTIQUES

VK BT

subordonnée complétive

\begin{tabular}{rrrrrrrl}
$\mathrm{v}$ & $\mathrm{d}$ & $\mathrm{c}$ & $\mathrm{c}_{t}$ & $\mathrm{v}$ & $\mathrm{d}$ & $\mathrm{c}$ & $\mathrm{c}_{t}$ \\
52 & 29 & 32 & 2 & 22 & 24 & 20 & 4 \\
58 & 28 & 37 & 21 & 17 & 5 & 39 & 5 \\
2 & 3 & 4 & - & - & - & 2 & - \\
28 & - & - & - & 4 & 2 & - & - \\
\hline 140 & 50 & 73 & 23 & 43 & 31 & 61 & 9 \\
286 & & & & 144 &
\end{tabular}

VK
$\mathrm{v}=49 \%$
$\mathrm{~d}=17 \%$
$\mathrm{c}=26 \%$
$\mathrm{c}_{t}=8 \%$

BT

$\mathrm{V}=30 \%$

$d=22 \%$

$\mathrm{d}=42 \%$

$c_{t}=6 \%$

LE TABLEAU D'APRÈS LES DOMAINES SÉMANTIQUES

domaine de la volonté

\begin{tabular}{rr} 
VK & BT \\
$49 \%$ & $30 \%$ \\
$17 \%$ & $22 \%$ \\
$26 \%$ & $22 \%$ \\
$8 \%$ & $6 \%$ \\
\hline $100 \%$ & $100 \%$
\end{tabular}


21. REVUE DE L'EMPLOI DU SUBJONCTIF DANS LE CORPUS EN ENTIER

Notre corpus dans son entier nous montre le tableau suivant:

$\begin{array}{lrrrr} & \text { MB } & \text { ES } & \text { VK } & \text { BT } \\ \text { domaine de la volonte } & 28 \% & 36 \% & 49 \% & 30 \% \\ \text { domaine du doute } & 51 \% & 43 \% & 17 \% & 22 \% \\ \text { domaine du commentaire } & 18 \% & 20 \% & 26 \% & 42 \% \\ \text { domaine du commentaire } & 3 \% & 1 \% & 8 \% & 6 \%\end{array}$

Dans les oeuvres consultées, on constate certaines divergences dans I'emploi du subjonctif; on constate I'emploi fréquent du subjonctif dans le domaine de la volonté dans la chronique de Vissoko et le pourcentage extrêmement élevé du subjonctif dans le domaine du doute chez Flaubert. Il faut souligner ici que 1'emploi du subjonctif plpf. à valeur hypothétique est fort fréquent dans les originaux franqais (MB 80 fois, ES 41 fois) et presque inexistant dans les traductions. Si 1'on élimine cet emploi du subjonctif (pour MB cela représente le 28 o de toutes les subordonnees subjonctives, pour ES le $18 \%$ ), les différences de pourcentage entre les oeuvres originales et les traductions ne sont plus surprenantes.

Les listes nous montrent aussi le pourcentage élevé des actions certaines (commentaire et commentaire à délimitation temporelle):

$\begin{array}{ccccc} & \text { MB } & \text { ES } & \text { VK } & \text { BT } \\ \text { action certaine } & 29 \div & 26 \div & 34 \div & 50 \%\end{array}$

Ce pourcentage montre clairement quelle partie de l'emploi du subjonctif francais est absolument négligé par des théories qui definissent le subjonctif comme le mode du doute, d'un concept imaginaire, de la non-participation à une situation, bref, du non-réel ou du non-réalisé. Ces theories englobent la quasi-totalité des domaines de l'emploi du subjonctif mais elles en laissent de côté un quart au moins.

22. APERÇU CONTRASTIF

L'origine de ce travail était le désir d'une recherche 
contrastive, voire la nécessité d'examiner les difficultés que rencontre le locuteur slovène (et pas seulement slovène) en apprenant le franqais. La première tâche de 1'analyse contrastive est d'étudier les moyens linguistiques par lesquels le paradigme verbal, appelé subjonctif, inconnu en slovène comme forme verbale spéciale, est rendue en slovène. Inversement, les expériences de la traduction peuvent-être utiles pour celui qui apprend le franfais et qui doit apprendre aussi l'emplo1 correct de ces formes verbales inconnues dans sa langue.

Aux propositions francaises avec le subjonctif correspondent en slovène les subordonnées complétives, adverbiales, relatives, les propositions indépendantes ou principales, l'imperatif pour la 3.ème personne, le plus-que-parfait du subjonctif, l'infinitif et aussi les éléments isolés de la proposition, comme les substantifs, les adjectifs et les adverbes.

La seule situation qui nous intéresse étant celle où, dans la traduction aussi, la forme verbale est conservee, nous constatons que dans les traductions en slovène les formes verbales sont les suivantes:

a) dans le domaine de la volonté l'indicatif, le conditionnel, l'optatif, l'imperatif;

b) dans le domaine du doute l'indicatif, le conditionnel, l'optatif rarement;

c) dans le domaine du commentaire et du commentaire à délimitation temporelle

presque exclusivement $I^{\prime}$ indicatif, très rarement le conditionnel.

23. Il est important de souligner que la structuration de cette réalité extralinguistique qui aura, dans la réalisation linguistique en français, le subjonctif, est assez analogue dans les deux langues analysees: $85 \%$ des cas ont la structure proposition principale - proposition dépendante. 15 \& seulement de toutes les phrases franqaises examinées ont en slovène une structure différente. Ces derniers cas, ne présentant ici aucun intérêt, on s'attachera à ceux où les deux langues, dans ce domaine grammatical concordent structurellement. La stati- 
stique montre que le subjonctif francais, comme il apparaît dans les quattre oeuvres litteraire de notre corpus, est présent ou rendu en slovène par

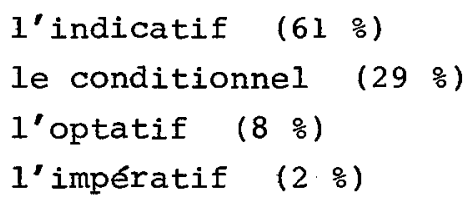

La langue francaise et slovène articulent donc l'espace du mode verbal d'une manière très différente. Le locuteur slovène qui apprend le francais fait connaissance avec une catégorie verbale inconnue dans sa langue et doit surtout apprendre les principes selon lesquels cette langue étrangère articule un secteur de la réalité extralinguistique; il ne suffit pas d'apprendre des termes dans la langue etrangère qui sectionnent cette réalité, ni d'apprendre tout simplement une nouvelle nomenclature, mécaniquement. Les fautes que commet un étranger sont précisément celles qui sont conditionnées par le système de sa propre langue.

23. L'incertitude des étrangers sur I'emploi du subjonctif est conditionné aussi par une présentation trop partielle de cette importante catégorie grammaticale.

Aucune grammaire, à une exception près, ne met pas en évidence le fait que le subjonctif n'est pas seulement le mode de l'incertitude, mais qu'il s'emploie aussi pour exprimer des faits certains. Au contraire, selon l'enseignements des grammaires le subjonctif est avant tout le mode de l'incertitude. Par contre, le corpus qu'on vient d'examiner présente a peu près un tiers des cas où le fait est conqu comme certain. Les grammaires parlent souvent du domaine des sentiments comme caractéristiques de l'emploi du subjonctif, mais sans indiquer que ces sentiments se rapportena à un fait réel.

Des enquêtes, menées auprès des étudiants slovènes, mont trent qu'ils ont peu de difficultés dans l'emploi correct du subjonctif dans le domaine de la volonté et du doute; par contre l'embarras surgit dans le domaine du commentaire et du com- 
mentaire a délimitation temporelle, c'est-à-dire la où ce paradigme verbal sert à exprimer la certitude.

c'est justement ce domaine qui exige une double attention de la part de celui qui apprend le francais et ne connaît pas, dans sa propre langue, un système de formes verbales analogue à celui du français: il est tenu d'assimiler le système de la langue qu'il veut apprendre.

Le travail que nous présentons n'a pas été conqu comme une simple recherche des procédés pédagogiques et méthodologiques qui devraient faciliter l'apprentissage d'un important phénomène $d$ 'une langue étrangère. On a cherché aussi a élaborer une typologie simple et exhaustive du subjonctif roman en se placant dans un angle visuel de la fonction d'un signe linguistique.

On a tenté de faire une description sémantique d'un phénomène linguistique (on sait combien l'emploi du subjonctif est particulierement délicat) en délimitant en même temps les principes structuraux qui permettent un classement fondé des propositions subjonctives d'après leur valeur sémantiques et $l^{\prime}$ analyse des critères $d^{\prime} u n$ tel classement. Cette étude a soumis à la critique les théries sémantiques plus articulées sur le subjonctif francais et elle en a démontré l'insuffisance. Pour analyser le subjonctif francais, elle a proposé de l'inclure dans les domaines sémantiques de volonté, du doute et du commentaire et enfin de le classifier à partir des critères structuraux de l'antériorité et de l'implication de la certitude de l'état réel d'un fait. 


\title{
OEUVRES LITTÉRAIRES CITÉES
}

\author{
FLAUBERT G.: $\quad$ Madame Bovary \\ 1961 (1857), Paris, Garnier, 467 pgs. \\ FLAUBERT G.: - Gospa Bovaryjeva \\ 1964, Ljubljana, Cankarjeva založba, 347 pgs. \\ FLAUBERT G.: L'Education sentimentale \\ 1964 (1869), Paris, Garnier, 473 pgs. \\ FLAUBERT G.: Vzgoja srca I-II \\ 1968, Ljubljana, Cankarjeva založba, $176+272$ \\ pgs. \\ TAVČAR I.: Visoška kronika \\ 1968 (1931), Ljubljana, Mladinska knjiga, 233 \\ pgs. \\ TAOUTCHAR I.: La Chronique de Vissoko \\ 1975, Paris, Publications Orientalistes de France, \\ 249 pgs., traduit par Jean Durand Monti et Viktor \\ Jesenik. \\ KOSMAC C.: Balada o trobenti in oblaku \\ 1977 (1964), Ijubljana, Mladinska knjiga, 189 \\ pgs. \\ KOSMAC C.: La Ballade de la trompette et du nuage \\ 1977, Paris, Publications Orientalistes de Fran- \\ ce, 165 pgs., traduit par Jean Durand Monti
}

OUVRAGES CONSULTÉS

(Bibliographie sélectionnée)

Grammaire de I'ACADÉMIE FRANÇAISE:

1932, Paris

ALLAIRE S.: $\quad$ Le syntagme LE FAIT QUE

1975, Le Français moderne 43, pgs. 308-337

ANDERSSON S.: $\quad$ Nouvelles études sur la syntaxe et la sémantique du mot francais TOUT 1961, Lund

ANDERSSON S.: L'emploi du subjonctif après un superlatif 1972, Moderna Språk 66, pgs. 156-161

AUSTIN J.: $\quad$ How to Do Things with Words 1962, Oxford, Oxford University Press

BADÍA MARGARIT A.: El subjuntivo de subordinación en las lenguas romances y especialmente en iberroromanico 1953, Revista de Filología española 37, pgs. 95129 
BALLY Ch.: Linguistique générale et linguistique française 1965 (4.), Berne, A. Francke, 44 pgs.

BARRAL M.: $\quad$ Remarques sur l'emploi des temps du subjonctif en francais moderne: valeurs temporelles et modales

1962, Revue des langues romanes LXXV, pgs. 39-78

BARRAL M.: L'imparfait du subjonctif dans la concordance des temps 1964, Le Français dans le monde 26, str. 12-15

BARRAL M.: La construction de APRÈS QUE avec le subjonctif en français 1967, Le Franfais dans le monde 50, pgs. 20-22

BARRAL M.: L'Imparfait du Subjonctif 1980 , Paris, Picard, 627 pgs.

LE BIDOIS G.-LE BIDOIS R.: Syntaxe du francais moderne I-II 1968, Paris, Auguste Picard, $560+794$ pgs.

de BOER C.: $\quad$ Syntaxe du francais moderne 2947, Leiden, Universitaire Pers Leiden, 352 pgs.

BOLEO M.: Tempos e modos em portugues 1934, Boletim de filologia III/1-2, pgs. 15-36

BRUNOT F.: $\quad$ La Pensée et la Langue 1953 (1926), Paris, Masson, 982 pgs.

BRUNOT F.-BRUNEAU C.: précis de grammaire historique de la langue franqaise 1969, Paris, Masson, 589 pgs.

CARLSSON L.: Le type C'EST LE MEILLEUR LIVRE QU'IL AIT JAMAIS ÉCRIT en espagnol, en italien et en franGais 1969, Uppsala, Acta Univ. Upsal., Studia Romanica Upsaliensia 5, 92 pgs.

CHRISTMANN H.: Zum Verhältnis zwischen dem italienischen und dem französischen Konjunktiv, Interlinguistica (Festschrift Wandruszka), 1971, Tübingen, pgs. 103-114

COHEN M.: $\quad$ Le subjonctif en françis contemporain 1965, Paris, Société d'enseignement supérieur, 294 pgs.

DAMOURETTE J.-PICHON E.: Des Mots à la Pensée V 1911-1936, Paris, Bibliothèque du "francais moderne", 861 pgs.

DAUZAT A.: $\quad$ Grammaire Raisonnée de la langue francaise 1947, Lyon, IAC, 481 pgs.

FOULET L.: $\quad$ Petite syntaxe de 1 'ancien français 1930, Paris, H. Champion, 393 pgs.

FREEMAN M.: $\quad$ Constraints on rejoinders: the case of the subjunctive in Romance 1974, (manuscrit) 21 pgs. 
GLOBEVNIK D.: Essai d'analyse sémantique des modalités verbales prédicatives dans un texte de G. Flaubert 1978, Linguistica XVIII, pgs. 217-236

GLOBEVNIK D.: Syntaxe et/ou sémantique a propos du subjonctif francais 1979, Linguistica XIX, pgs. 223-242

GLOBEVNIK D.: Les modalités verbales en français: théorie fonctionnelle de la langue

1981, La Linguistique 17/I, pgs. 113-126

GOUGENHEIM G.: Système grammatical de la langue franqaise 1938, Paris, D'Artrey, 373 pgs.

GREVISSE M.: Le Bon Usage 1964, Gembloux-Paris, Duculot-Hatier, 1194 pgs.

GUILLAUME G.: Temps et verbe - Theorie des aspects, des modes et des temps 1929, Paris, H. Champion, 134 pgs.

HAAS J.: Neufranzösische Syntax 1909, Halle (Saale), Max Niemeyer, 493 pgs.

HALL R. jun.: Il congiuntivo indipendente 1974, Studi di Grammatica Italiana 4, pgs. 109114

HANSE J.: La valeur modale du subjonctif 1960, Bulletin de 1'Académie Royale de Langue et de Littérature Française XXXVIII, pgs. 107130

IMBS P.: Le subjonctif en francais moderne 1953, Strasbourg, Publications de la faculté des lettres, pgs. 70

KALEPKY Th.: Neuaufbau der Grammatik 1928, Leipzig

KIPARSKY P.: Tense and Mood in Indo-European Syntax 1968, Foundations of Language 4, pgs. 30-57

KLUM A.: Rapports temporels, modes d'action et problèmes connexes 1975, Studia.neophilologica $47 / 2$, pgs. 213-241

LERCH E.: Die Bedeutung der Modi im Französischen 1919, Leipzig, Reisland, 111 pgs.

LEVITT J.: The Subjonctive in Modern French: Conflicts between Theory and Usage 1967, Linguistics 31, pgs. 50-60

MARTINET A.: Grammaire fonctionnelle du francais 1979, Paris, Didier, 276 pgs.

MAUGER G.: $\quad$ Grammaire pratique du francais d'aujourd'hui 1968, Paris, Hachette, 416 pgs.

MOIGNET G.: $\quad$ Essai sur le mode subjonctif I-II 1959, Paris, Presses Universitaires de France, 756 pgs. 
van der MOLEN W.: Le subjonctif: sa valeur psychologique et son emploi dans la langue parlée, 1923 Amsterdam

NORDAHL H.: Les systemes du subjonctif corrélatif

1969, Bergen-Oslo, Universitetsforlaget, $271 \mathrm{pgs.}$

PEIXOTO DA FONSECA F.: O emprego do conjuntivo em portugues contemporaneo

1968, Actele celui de-al XII lea congres International de Linvistică şi Filologie Romanică, Bukarešta, pgs. 475-480

de POERCK G.: Modalite et modes en francais

1950, Le Francais moderne 18, pgs. 171-188

POHL J.: $\quad$ Forme et Pensée

S. a., Paris, Wesmael-Charlier

RAT M.: $\quad$ Grammaire francaise pour tous

1968, Paris, Garnier

REGULA J.: $\quad$ Grammaire françise explicative

1957, Heidelberg. C. Winter, 244 pgs.

RONSJÖ E.: Le mode du verbe dans les propositions complétives introduites par LE FAIT QUE, 1966

ROTHE W.: $\quad$ Strukturen des Konjunktivs im Französischen 1967, Tübingen, M. Niemeyer, 427 pgs.

RUWET N. Introduction à la grammaire generative 1967, Paris, Plon, 452 pgs.

SCHIFKO P.: Subjonctif und Subjuntivo

1967, Wien, W. Braumüller, 217 pgs.

SCHMITT JENSEN J.: Subjonctif et hypotaxe en italien

1970, Odense, Odense University Press, 748 pgs.

SCHOGT H.: Le système verbal du françis contemporain

1968, Hague-Paris, Mouton, 74 pgs.

SOLTMANN H.: $\quad$ Syntax der Modi im modernen Französisch

1914, Halle (Saale), M. Niemeyer, 266 pgs.

SVANE G.: $\quad$ Grammatik der Slowenischen Schriftsprache

TESNIÈRE L.: Elements de syntaxe structurale

1966, Paris, Klincksieck, 670 pgs.

TOGEBY $K_{.}: \quad$ Mode, aspect et temps en espagnol

1953, Kфbenhavn, Munksgaard, 136 pgs.

ROGEBY K.: $\quad$ Structure immanente de la langue francaise

1965, Paris, Larousse, 208 pgs.

TOPORIŠIC J.: Slovenska slovnica

1976, Maribor, Obzorja, 588 pgs.

VINCENOT C.: Essai de grammaire slovène

1975, Ljubljana, Mladinska knjiga, 353 pgs. 
WANDRUSZKA M.: Sprachen - Vergleichbar und Unvergleichbar 1969, München

von WARTBURG W.-ZUMTHOR P.: Précis de syntaxe du français contemporain

1947, Berne, A. Francke, 356 pgs.

\author{
Povzetek \\ KONJUNKTIVNE GLAGOLSKE MODALNOSTI V LUČI FUNKCIONALNE \\ KONTRASTIVNE FRANCOSKO-SLOVENSKE ANALIZE
}

\begin{abstract}
Filozofska fakulteta $\nabla$ Ljubljani je 20. oktobra 1980 sprejela temo "Konjunktivne glagolske modalnosti $v$ luči funkcionalne kontrastivne francosko-slovenske analize", ki jo je predlagala kot temo doktorske disertacije mag. Darja Globevnikova, tedaj asistentka pri SAZU, Razredu za literarne in jezikoslovne vede, in me izbrala za mentorja. Mag. Globevnikova je v februarju 1983 svoje delo (VIII+ 313 str.) dokončala in ga predložila fakulteti $v$ oceno. V marcu 1983 pa se je tragično končalo njeno żivljenje in znanstveno delo. Tako ni prišlo do javne obrambe disertacije.

Da pa bi bili izsledki većletnega temeljitega dela in teoretične postavke, na katerih je doktorandka gradila, vendar dostop$n i$ strokovni javnosti, zlasti še romanistom, $k i j i h$ sintaksa romanskega glagola in naklona $v$ francoščini posebej privlači, objavlja Linguistica strnjen povzetek disertacije v letniku, ki je v celem posvečen objavi prvih del mlade znanstvene generacije na Filozofski fakulteti v Ljubljani.

Ko sem pripravljal tekst za objavo, je bila moja glavna skrb, da kljub nujnemu krajšanju kolikor mogoče verno podam teoretično zasnovo dela in avtoričine izsledke. - Mitja skubic
\end{abstract}

Francoski in nasploh romanski glagol ima v bogastvu svojih paradigem tudi vrsto oblik, ki jih imenujemo konjunktiv. Iz praktičnih izkušenj vemo za težave, ki jih ima vsakdo z uporabo teh glagolskih paradigem, zlasti še, kdor v svojem jeziku takih paradigem ne pozna. Očitek, ki ga je mogoče izreči slovnicam francoskega jezika in specifičnim delom, ki se ukvarjajo z romanskim glagolskim naklonom, je predvsem ta, da skušajo potegniti na skupni imenovalec vso široko uporabo teh glagolskih oblik, in ta skupni imenovalec je največkrat dvom, negotovost, želja, skratka zmeraj neko nerealizirano dejanje. V strokovni literaturi je moč najti na semantiki zasnovane teorije, ki izhajajo iz dejstva, da gre pri konjunktivu za jezikovni izraz v Saussurovem smislu, da imamo torej opraviti s psihično podstavo, za katero iščemo glasovni izraz. Rdeča nit, ki se vleče skozi vso disertacijo, je prav ta, da bi določili psihično podstavo, ki je vodila kizbiri posebnih glagolskih oblik, saj nikakor niso zadovoljive tiste semantične teorije, ki skušajo videti $v$ konjunktivu samo izraz osebnega mnenja in subjektivne sudbe, torej izraz za nerealizirano dejanje. V analiziranih literar:il delih pa najdemo vsaj tretjino primerov, ko gre za izvrsena dejanja. Se manj so prepričljive tiste teorije, ki vidijo $v$ konjunktivu samo sintaktićno 
varianto indikativa, in torej puščjo vnemar semantično komponento, čeprav so potem prisiljene navesti vrsto glagolov, $k i$ "zahtevajo" konjunktivno obliko glagola v odvisniku.

Disertacija skuša zajeti pomensko vrednost, funkcionalnost konjuntiva in njegovo skladenjsko vezavo v tri pomenska območja, ki so volja, dvom, komentar. Pri določanju teh treh pomenskih območij si disertacija pomaga z dvema pomensko-skladenjskima testoma, in sicer s testom pomenske implikacije, kjer s pomočjo izsečka ugotavlja, ali je vodvisniku zaobjeta misel o realizaciji dejanja, in s testom preddobnosti, s čimer ugotavlja, ali lje vodvisniku izraženo dejanje lahko preddobno. V območju voje taka preddobnost ni mogoča, saj ni moč vplivati na pretekle dogodke ali ukazovati "za nazaj": volja ni retrospektivna. Realizacija nekega dejanja pa ni zaobjeta ne v območju želje ne v. obmocju dvoma. obmoćje komentarja pa dopušca preddobnost, obenem pa predstavija dejanje kot neko dejstvo, čeprav gledano skozi prizmo občutka ali psihičnega stanja: vesel sem, da pride naznanja, da bo dejanje realizirano in tako dejanje dopušca preddobnost: vesel sem, da je prišel.

Analitična shema bi ponazorila stavke s konjunktivom takole:

volja implikacija realizacije preddobnost

dvom

komentar

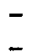

zunaj te tabele ostaja le nekaj primerov, predvsem tistih, $k i$ bi jih lahko uvrstili v komentar, vendar jih natančneje določa že kako prislovno določilo časa.

Disertacija je zasnovana kontrastivno, in sicer so vzeti primeri iz dveh francoskih in dveh slovenskih literarnih del (Flaubert, Tavčar, Kosmać) ter njihovih ustreznih prevodov in izhaja iz težav, ki jih ima slovenec, pa seveda ne samo ta, ko se uči romanskega jezika, z uporabo konjunktiva: usvojiti si mora drugačen sistem. Ni namreč zelo pomembno, da se kdo spozna s terminologijo sistemskih prvin v tujem jeziku: obvladati mora sistem. Slovenščina nima posebnih glagolskih paradigem za pojme, ki jih $v$ romanskih jezikih izraža konjunktiv. Vseeno velja povedati, da je primerjava s francošcino koristna: v analiziranih literarnih delih se $v$ francoščini konjunktiv pojavlja $v 85$ \% $v$ odvisniku, ki ima ob sebi glavni stavek. Táko poved poznata tudi prevoda $v$ slovenšcino, oziroma obe originalni deli in manj kot desetina pa je primerov, kjer ima francoski odvisnik s konjunktivom $v$ slovenščini ustreznico, ki ni glagol. Seveda skuša slovenščina izraziti pojem negotovosti, želje, osebne sodbe, dvoma s kakim prislovom: to pa omogoča slovenščini, da ob takem prislovu uporabi različne naklone: v izbranem korpusu je $61 \%$ francoskih konjunktivov prevedenih v slovenšcino s povednim naklonom, $29 \%$ s pogojnikom, $8 \%$ z želelnikom in 2 \% velelnikom. Francošc̆ina in slovenšc̆ina razc̆lenjujeta torej vsaka po svoje, z drugačnimi jezikovnimi sredstvi zunajjezikovni pojmovni prostor, ki ga v francoščini izraža konjunktiv. Težave za tujca so torej na diani, saj prevajamo po smislu, ne pa besedí ali struktur. Izvirajo pa tudi iz enostranskosti predstavitev konjunktiva $v$ francoscini: $v$ vseh slovnicah se trdi, da je konjunktiv sredstvo za izraz nerealiziranega dejanja, nečesa dvomlji- 
vega, a statistična analiza uporabljenih literarnih del kaže, da je nekako tretjina primerov takih, da je izraženo realizirano dejanje. Zato ni presenetljivo, da je pokazala anketa med študenti francošc̆ine, kako ti nimajo prav nobenih težav za pravilno uporabo francoskega konjunktiva $v$ območju volje ali dvoma, da pa nastopijo težavo takoj, ko je mogoče dejanje zajeti v obmocjje komentarja. Slovnice na take primere ne opozarjajo, in sploh ne ugotavljajo, da bi bila uporaba konjunktiva mogoča tudi zunaj nerealiziranega, samo domnevnega, umišljenega dejanja. $\checkmark$ veliki meri so bile prav težave tujca, ki v svojem jeziku nima glagolskih oblik, ustreznih romanskemu konjunktivu, tiste, $k i$ so narekovale delitev na pomenska območja volje, dvoma in komentarja, kar naj bi, tudi s pomočjo strukturalnih kriterijev, bolje osvetlilo psihično podstavo, za izraz katere ima francošcina posebno glagolsko paradigmo, konjunktiv. 